\title{
Estimation of energy budget of ionosphere-thermosphere system during two CIR-HSS events: observations and modeling
}

\author{
Olga Verkhoglyadova ${ }^{1, *}$, Xing Meng ${ }^{1}$, Anthony J. Mannucci ${ }^{1}$, Bruce T. Tsurutani ${ }^{1}$, Linda A. Hunt ${ }^{2}$, \\ Martin G. Mlynczak ${ }^{3}$, Rajkumar Hajra ${ }^{4}$, and Barbara A. Emery ${ }^{5}$ \\ 1 Jet Propulsion Laboratory, California Institute of Technology, Pasadena, CA 91109, USA \\ *Corresponding author: Olga.Verkhoglyadova@jpl.nasa.gov \\ 2 Sciences Systems and Applications, Inc., Hampton, VA 23681, USA \\ 3 NASA Langley Research Center, Hampton, VA 23681, USA \\ 4 Instituto Nacional de Pesquisas Espaciais, Sao Jose dos Campos, Sao Paulo, 12227-010, Brazil \\ 5 HAO/NCAR, Boulder, CO 80301, USA
}

Received 15 June 2015 / Accepted 14 March 2016

\begin{abstract}
We analyze the energy budget of the ionosphere-thermosphere (IT) system during two High-Speed Streams (HSSs) on 22-31 January, 2007 (in the descending phase of solar cycle 23) and 25 April-2 May, 2011 (in the ascending phase of solar cycle 24) to understand typical features, similarities, and differences in magnetosphere-ionosphere-thermosphere (IT) coupling during HSS geomagnetic activity. We focus on the solar wind energy input into the magnetosphere (by using coupling functions) and energy partitioning within the IT system during these intervals. The Joule heating is estimated empirically. Hemispheric power is estimated based on satellite measurements. We utilize observations from TIMED/SABER (Thermosphere-IonosphereMesosphere Energetics and Dynamics/Sounding of the Atmosphere using Broadband Emission Radiometry) to estimate nitric oxide (NO) and carbon dioxide $\left(\mathrm{CO}_{2}\right)$ cooling emission fluxes. We perform a detailed modeling study of these two similar HSS events with the Global Ionosphere-Thermosphere Model (GITM) and different external driving inputs to understand the IT response and to address how well the model reproduces the energy transport. GITM is run in a mode with forecastable inputs. It is shown that the model captures the main features of the energy coupling, but underestimates NO cooling and auroral heating in high latitudes. Lower thermospheric forcing at $100 \mathrm{~km}$ altitude is important for correct energy balance of the IT system. We discuss challenges for a physics-based general forecasting approach in modeling the energy budget of moderate IT storms caused by HSSs.
\end{abstract}

Key words. Ionosphere (general) - Thermosphere - Energy distribution - Solar wind - Modelling

\section{Introduction}

High-speed streams (HSSs) and associated corotating interaction regions (CIRs) are typical solar wind causes of moderate geomagnetic activity especially during the descending phase of a solar cycle (Tsurutani et al. 1995, 2006). Estimates of the energy and momentum inputs to the ionosphere-thermosphere (IT) system through interaction of the solar wind with the Earth magnetosphere have been of major interest (e.g., Knipp et al. 2005; Lu 2006; Mlynczak et al. 2008; Turner et al. 2009; Mlynczak et al. 2010a, 2010b; Deng et al. 2011; Hajra et al. 2014). It has been shown that time-integrated energy input into the IT system during a long-duration HSS interval can be larger than that during a typical CME-type storm (Tsurutani et al. 1995; Guarnieri 2006; Kozyra et al. 2006; Turner et al. 2009; Knipp et al. 2011; Verkhoglyadova et al. 2011). CIR-HSS storms have a strong impact on the IT system even though geomagnetic activity is moderate (Burke et al. 2010; Burns et al. 2012). The focus of this paper is to quantify the energy transport from the solar wind into the IT system during two HSS events. The study will combine empirical estimates and satellite measurements of proxies for energy input and modeling efforts.

We will analyze external driving of the IT system during two CIR-HSS sub-intervals, 22-31 January, 2007 (in the descending phase of solar cycle 23) and 25 April-2 May, 2011 (in the ascending phase of solar cycle 24). By studying two similar CIR-HSS events we aim to understand differences and similarities in the magnetosphere-IT coupling caused by similar external driving.

External energy input into the IT system occurs through many channels, including solar EUV (extreme ultraviolet) radiation, Joule and frictional heating (associated with increases in field-aligned current (FAC), ion-neutral collisions, and downward Poynting flux), and particle precipitation (traditionally considered mainly in the vicinity of the auroral oval). A comprehensive overview of the major energy and momentum inputs into the IT can be found in Heelis (2013). Solar wind coupling functions derived from interplanetary parameters are used as proxies of magnetospheric energy input during magnetic reconnection at the dayside magnetopause (see a brief overview in Newell et al. 2007). We will discuss energy partitioning in the IT system, namely distribution of energy among different physical mechanisms. It has been shown that the ring current contribution to the total IT energy budget during HSS-type storms is small $(\sim 10 \%)$ in comparison to Joule heating $(>60 \%)$ and particle precipitation ( 20\%) (Turner et al. 2009; Hajra et al. 2014). We will first address Joule heating and later discuss auroral heating due to particle precipitation (mostly magnetospheric electrons). 
Thayer \& Semeter (2004) showed that during geomagnetic activity intervals the total electromagnetic energy flux in the high-latitude ionosphere below $200 \mathrm{~km}$ substantially exceeds the total solar EUV flux. It follows from the Poynting theorem for long-term averages (in a steady-state approximation) that the Poynting flux $(\boldsymbol{S})$ divergence causes Joule heating and changes in the mechanical energy of the IT system (Lu et al. 1995; Thayer et al. 1995; Richmond 2010):

$$
-\nabla \cdot \boldsymbol{S}=\boldsymbol{j} \cdot \boldsymbol{E}+\boldsymbol{V}_{\mathrm{n}} \cdot \boldsymbol{j} \times \boldsymbol{B}, \quad \boldsymbol{j}=\sigma_{\mathrm{P}} \boldsymbol{E}
$$

where $\boldsymbol{j}$ is the current, $\boldsymbol{V}_{\mathrm{n}}$ is the neutral wind velocity, $\boldsymbol{B}$ is the magnetic field, $\boldsymbol{E}$ is the electric field in the inertial frame, and $\sigma_{\mathrm{P}}$ is the Pedersen conductivity. Joule heating accounts for IT heating due to electric current dissipation (e.g., Cole 1961). Joule heating can be determined either from the right-hand part of the Eq. (1), which is the electromagnetic energy deposition rate by definition, or through $\boldsymbol{S}$ directly with highlatitude electric and magnetic field potential patterns (Weimer 2005). Note that the electromagnetic energy deposition rate is not equal to the Joule heating and can deviate from it substantially, especially above $120 \mathrm{~km}$ altitude due to a contribution from neutral winds (Thayer \& Semeter 2004). However, height-integrated Joule heating is modeled to be $\sim 94 \%$ of the total energy deposition ( $\mathrm{Lu}$ et al. 1995). Joule heating is difficult to estimate from observations. It is dependent on conductivity and ultimately on electron density, which changes throughout a geomagnetic storm. Deng \& Ridley (2007) have shown numerically that Joule heating affects atmospheric density, electron density, and changes composition, and these effects depend on local time and altitude. Thus, thermosphere-ionosphere coupling is very important for an accurate estimation of IT heating. In this paper both empirical and self-consistent numerical estimates of the Joule heating and empirical polar convection patterns will be analyzed for the two HSS intervals.

Cooling radiation from nitric oxide (NO) is an important instantaneous proxy for geomagnetic storm energy input into the thermosphere (above $\sim 100 \mathrm{~km}$ altitude) and is suited for empirical estimation of Joule heating during HSS intervals because it accounts for up to $\sim 80 \%$ of the Joule heating enhancement (Mlynczak et al. 2003, 2010b; Lu et al. 2010; Verkhoglyadova et al. 2011, 2013, 2015). We use measurements of infrared emission from NO molecules at $5.3 \mu \mathrm{m}$ with the SABER (Sounding of the Atmosphere using Broadband Emission Radiometry) instrument on board the TIMED (Thermosphere-Ionosphere-Mesosphere Energetics and Dynamics) satellite (Mlynczak et al. 2010a). Infrared cooling emission from carbon dioxide $\left(\mathrm{CO}_{2}\right)$ molecules at $15 \mu \mathrm{m}$ governs radiative cooling up to $\sim 140 \mathrm{~km}$ altitude (Mlynczak et al. 2010a). In this paper we will use estimates of zonal cooling radiation fluxes in several altitude ranges and global daily powers based on SABER measurements.

Auroral heating is assumed to be due to electrons of $<30 \mathrm{keV}$ energy range precipitating into the vicinity of the auroral oval. Auroral heating typically contributes up to $\sim 22 \%$ of the total energy balance in the IT system during HSS events (Turner et al. 2009; Hajra et al. 2014). In this paper auroral electron power or the electron hemispheric power (HPe) is estimated from inter-calibrated NOAA POES (Polar Orbiting Environmental Satellite) and DMSP (Defense Meteorological Satellite Program) datasets (Emery et al. 2006, 2008).

The hemispheric power index (HPI) is based on the total energy flux from ions and electrons ( $\mathrm{HPt}$ ) of $<20 \mathrm{keV}$ energy range from NOAA satellites, and is organized in terms of increasing $\log$ power. It is written as $\mathrm{HPI}=2.09 \times \ln (\mathrm{HPt})$ or $\mathrm{HPt}=4.2^{(\mathrm{HPI} / 3)}$ (Maeda et al. 1989; Emery et al. 2006, 2008, 2012). Foster et al. (1986) listed 9 HPI patterns of energy flux and mean energy from NOAA POES satellites associated with certain $K p$ ranges. Since ions with energies $<20 \mathrm{keV}$ only contribute $\sim 5-20 \%$ (Emery et al. 2006, 2008) of the total energy flux with increasing percentages for quiet conditions, these HPI patterns can be considered as caused primarily by electrons and approximated by HPe. All available electron precipitation data from NOAA POES $(<20 \mathrm{keV})$ and DMSP $(<30 \mathrm{keV})$ satellites were compared and inter-calibrated by Emery et al. (2006, 2008, 2009, 2011), where the inter-satellite relations were changed according to revised calibrations (e.g., Hardy et al. 2008). HPe from both hemispheres was assumed to be the same over the lifetime of each satellite, which was considered to be a long enough period to account for the clear seasonal differences in HPe (Emery et al. 2006, 2008). Hourly estimates of HPe are available online from http:// cedarweb.vsp.ucar.edu/wiki/index.php/Tools_and_Models:Emery_ HP_plus_indices_to_11107.

Another question that this paper addresses is how well modeling captures energy transport based on our current understanding of underlying physical processes. We perform modeling of these two HSS intervals using the Global Ionosphere-Thermosphere Model (GITM) as the reference model (Ridley et al. 2006), run in a forecasting mode, i.e., with forecastable inputs and without event tuning. We will estimate and analyze IT energy partitioning, Joule heating, $\mathrm{NO}$ and $\mathrm{CO}_{2}$ cooling, and auroral heating throughout the HSS intervals and make comparisons with empirical estimates.

The second section of the paper will briefly introduce the interplanetary conditions for these two HSS events and present empirical energy proxies and estimates for external driving of IT. The third section will describe the modeling approach, discuss drivers, and present numerical estimates for the energy budget. We will analyze results in Section 4. This study is an extension of previous studies on energy transport and partitioning in HSS events (Verkhoglyadova et al. 2011, 2015) with the use of physics-based modeling. We will discuss forecasting challenges in Section 5.

\section{Observations and empirical estimations of external driving during two HSS intervals}

In this paper we will analyze CIR-HSS sub-intervals within the 22-31 January, 2007 and 25 April-2 May, 2011 intervals. The average sunspot numbers for these intervals were low, below 25 and below 50 for the first and second intervals, respectively. The geomagnetic storm index SYM-H had peak intensities of $-54 \mathrm{nT}$ and $-55 \mathrm{nT}$ for the two intervals. It is known that solar wind - magnetosphere - IT coupling is increased if the interplanetary magnetic field (IMF) has a southward component. It has been shown that a strong geomagnetic storm commences if the IMF southward component is $\geq 10 \mathrm{nT}$ at least $3 \mathrm{~h}$ (Gonzalez \& Tsurutani 1987), for which condition for the dawn-to-dusk geoeffective interplanetary electric field (Ey) translates into $E y \geq 5 \mathrm{mV} / \mathrm{m}$ (for a solar wind speed of $500 \mathrm{~km} / \mathrm{s})$. For moderate geomagnetic activity $(-100 \mathrm{nT} \leq$ $D s t \leq-50 \mathrm{nT})$, the criterion relaxes to $E y \geq 2 \mathrm{mV} / \mathrm{m}$ for intervals longer than $2 \mathrm{~h}$ (Echer et al. 2013). High-Intensity Long-Duration Continuous Auroral Activity (HILDCAA) is often observed during a HSS (Tsurutani \& Gonzalez 1987; Hajra et al. 2013) and plays an important role in the energy 
budget of a geomagnetic storm (Gonzalez et al. 2006; Hajra et al. 2014) and the IT system (Burke et al. 2010).

Below we specify which empirical parameters will be used in analysis of the events. Coupling functions are a way of quantifying the degree of interaction between the solar wind and the magnetosphere. They are derived from interplanetary parameters estimated at $1 \mathrm{AU}$ in front of the Earth's magnetopause. The epsilon function ( $\varepsilon$ : Perreault \& Akasofu 1978; Akasofu 1979) was originally derived for estimating the energy consumed in the inner magnetosphere only. There are known issues with scaling of the epsilon parameter (Koskinen \& Tanskanen 2002) and parametrizing the effective crosssectional area of the solar wind - magnetosphere interaction (Burke et al. 1999). However, the index is still the most widely used simple estimate of total energy input into the Earth's magnetosphere, even with certain underestimations. Traditionally, the epsilon parameter is assumed to account for the total energy budget with contributions from the ring current, Joule heating, and particle precipitation (Turner et al. 2009; Hajra et al. 2014). However, the total amount of energy in the IT system includes other important external energy inputs, e.g., neutral wind dynamo and chemical heating.

The Universal function (Newell et al. 2007) has a clear physical meaning: it estimates the rate of magnetic flux opened at the magnetopause. It is composed of terms accounting for the rate of IMF field lines merging at the dayside magnetopause, the fraction of IMF lines that merge, IMF magnitude, and merging field line length. This function is shown to correlate the best with activity indices (aside from $D s t$ ) at an hourly cadence (Newell et al. 2007). The Kan-Lee electric field is another coupling parameter derived from geometrical considerations for the dayside magnetopause reconnection (Kan \& Lee 1979). It accounts for reconnection of parallel field lines pertinent to high latitudes or cusp area.

Joule heating power is estimated from empirical formulae proposed by Knipp et al. (2004) for the northern hemisphere. We use the "winter" set of fit coefficients for the 2007 event and the "summer" set of fit coefficients for the 2011 event. These two sets account for seasonal changes in high-latitude ionospheric conductivity. Empirical Joule heating is determined by the Polar Cap (PC) index and Dst index taken from the OMNI dataset (http://omniweb.gsfc.nasa.gov/form/omni_ min.html). Here, the PC index is a proxy for the electric field imposed on the polar ionosphere by the solar wind and Dst is a proxy for the total ring current particle kinetic energy (electromagnetic interaction between the solar wind and mid-latitude ionosphere). We note that the estimated (northern hemisphere) Joule heating is a large-scale global parameter that does not include small-scale variability.

We will use the NO cooling radiation power as an independent proxy for the amount of geomagnetic energy input into the IT. The limb radiance profiles of NO emissions from SABER measurements are used to derive vertical profiles of the volume emission rate of energy $\left(\mathrm{W} / \mathrm{m}^{3}\right.$ ) (Mlynczak et al. 2010a). The vertical profiles are then integrated vertically (within 100 $250 \mathrm{~km}$ altitude) to obtain fluxes $\left(\mathrm{W} / \mathrm{m}^{2}\right)$. Further integration over all latitudes and longitudes for all scans per day yields the "measured" daily global power. We use the global power which is derived from the "measured" global power by interpolating from the highest latitudes of SABER scans to the poles.

The low-energy $(<30 \mathrm{keV})$ electron precipitation is described with the estimated HPe based on inter-calibrated measurements with NOAA POES and DMSP satellites to provide consistent estimates (Emery et al. 2006, 2008, 2009, 2011). The NOAA satellites offer good daytime coverage of the northern auroral hemisphere and good nighttime auroral coverage in the southern hemisphere. The DMSP satellites also preferentially cover the southern hemispheric nighttime aurora. Thus, the southern hemisphere results are generally more accurate than the northern hemisphere results.

In the next sub-sections we briefly describe interplanetary parameters and geomagnetic activity for the two HSS events. Empirical estimates for different channels of external energy input into the IT and energy partitioning will be provided.

\subsection{HSS sub-interval of 22-31 January, 2007}

Figure 1 shows interplanetary parameters for the event, the solar wind speed (a) and southward component of the interplanetary magnetic field, $B_{z}$ (b). Vertical lines approximately denote sample times during: pre-event background ( 00:00 UT on 24 January), the storm main phase ( 7:10 UT on 29 January, $\sim 14: 00$ UT on 29 January), and recovery phase ( 4:50 on 31 January), to be discussed later. Note that the IMF stays northward $\left(B_{z}>0\right)$ during the main phase. The HSS event starts at the beginning of January 29. The solar wind speed increases from the nominal slow solar wind value of $\sim 400 \mathrm{~km} / \mathrm{s}$ to almost $800 \mathrm{~km} / \mathrm{s}$. From a simultaneous sign change in IMF $B_{x}$ and IMF $B_{y}$ right at the beginning of the HSS we conclude that a heliospheric current sheet (HCS) crossing occurred (not shown here). The CIR signature is seen in large increases of IMF magnitude and plasma density (Smith \& Wolfe 1976; not shown), and abrupt large variations of $B_{z}$ at the leading edge of the HSS (panel b). Note more short-scale variations in $B_{z}$ during the HSS proper as compared with the pre-event background. Figure $1 \mathrm{k}$ presents the pressure-corrected high-resolution SYM-H index (Burton et al. 1975; Gonzalez et al. 1994; Katus \& Liemohn 2013). There is a negative SYM-H bay observed at the beginning of the CIR encounter. It is followed by a sudden decrease during the geomagnetic storm main phase. The HSS proper corresponds to a gradual storm recovery seen in SYM-H. Figure 11 shows the AE index which increases throughout the main phase of the storm and stays elevated during the recovery phase. The interval of intense auroral activity is characterized by peak and average AE intensities of $1494 \mathrm{nT}$ and $392 \mathrm{nT}$, respectively. The high AE activity continued from 14:02 UT of 29 January to $14: 14$ UT of 31 January, with a duration of $\sim 48.2 \mathrm{~h}$. The interval is well correlated with the HSS event and is characterized by Alfvénic waves (Hajra et al. 2014). AE variation follows the "HILDCAA criteria" suggested by Tsurutani \& Gonzalez (1987).

The total energy input into the magnetosphere-IT system as approximated by the epsilon parameter (Fig. 1c) shows an increase to $\sim 4000 \mathrm{GW}$ at the onset of the geomagnetic storm and sharply declines in the middle of the storm main phase. The epsilon parameter starts to grow during the "negative bay" interval. The Universal coupling function is shown in Figure 1d. It also increases with the geomagnetic storm onset, but stays elevated throughout the recovery phase, capturing a prolonged external driving possibly associated with HILDCAA. The Kan-Lee electric field is shown in Figure 1e. It is highly variable with the highest peaks observed throughout the main phase. However, the electric field of the recovery phase is still higher than that of the pre-event background, which indicates storm-time energy transfer. Joule heating for 


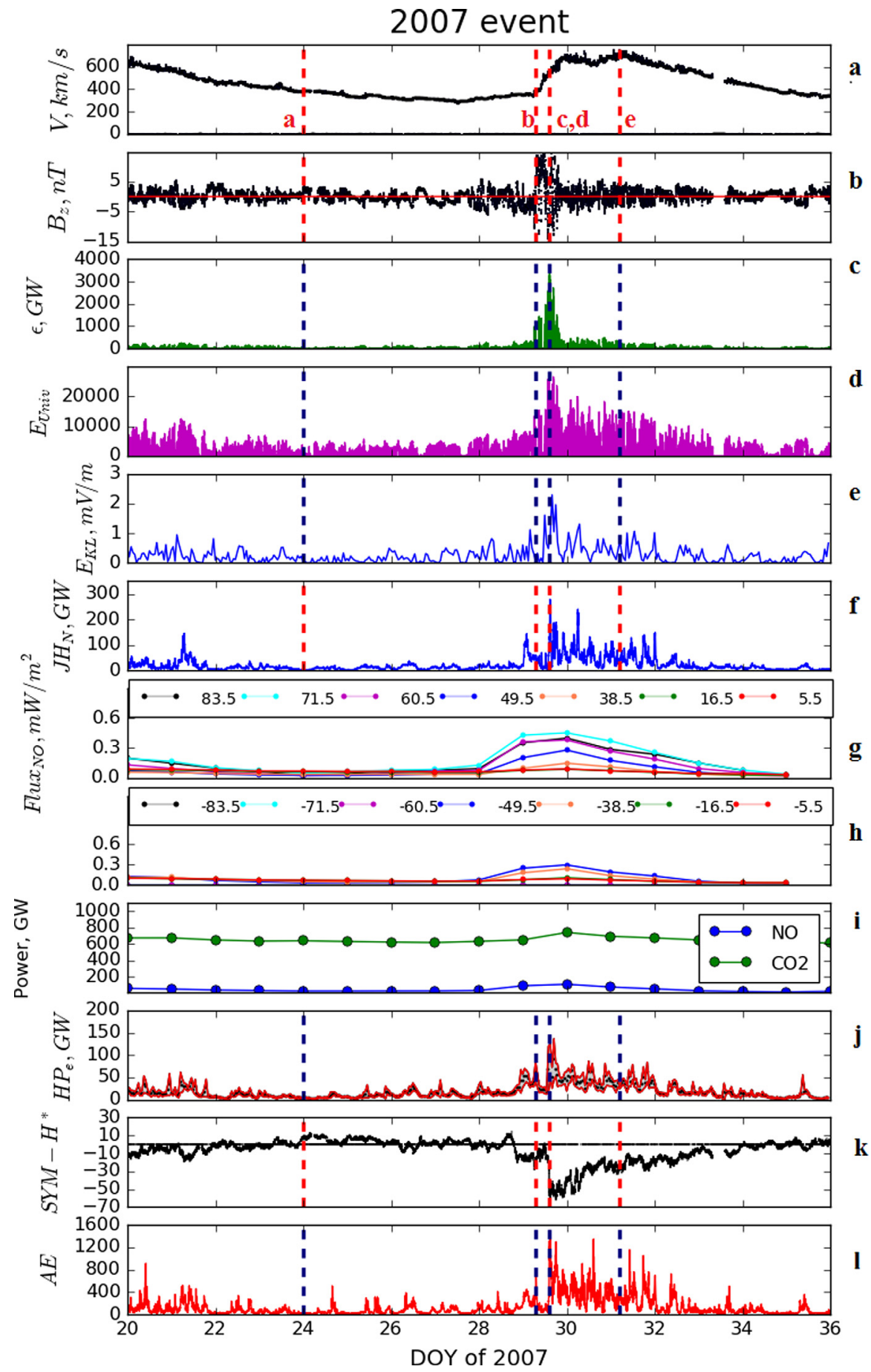

Fig. 1. Interplanetary parameters and coupling functions for the 2007 CIR-HSS event: (a) solar wind speed; (b) southward component of the interplanetary magnetic field $B_{z}$, (c) epsilon parameter; (d) universal coupling function; (e) Kan-Lee electric field. Joule heating estimated for the northern hemisphere is shown in panel (f). Daily thermospheric cooling fluxes computed from height-integrated NO emission profiles averaged in seven latitude bins corresponding to the central geographic latitudes of $5.5^{\circ}, 16.5^{\circ}, 38.5^{\circ}, 49.5^{\circ}, 60.5^{\circ}, 71.5^{\circ}$, and $83.5^{\circ}$ are shown in panel (g) for the northern hemisphere. The same parameters for the latitude bins corresponding to the central geographic latitudes of $-83.5^{\circ}$, $-71.5^{\circ},-60.5^{\circ},-49.5^{\circ},-38.5^{\circ},-16.5^{\circ}$, and $-5.5^{\circ}$ in the southern hemisphere are shown in panel (h). Panel (i) shows daily-averaged global cooling powers for $\mathrm{NO}$ and $\mathrm{CO}_{2}$. Electron hemispheric power, HPe (black dots) with standard deviation values (red bounding lines) from intercalibrated NOAA POES and DMSP measurements are shown in panel (j). Panels (k) and (l) show pressure-corrected SYM-H and AE indices, correspondingly. Vertical dashed lines indicate sample times during: pre-event background ( 00:00 UT on 24 January, snapshot a), the storm main phase ( 7:10 UT on 29 January, snapshot b; 14:00 UT and 15:00 UT on 29 January, snapshots $\mathrm{c}$ and d), and recovery phase ( 4:50 on 31 January, snapshot e). Snapshot numbering is shown in panel a. See text for details.

the northern hemisphere (Fig. 1f) closely resembles the Kan-Lee electric field dynamics. The peak Joule heating value is about $5 \%$ of the total energy input into the magnetosphere based on the epsilon parameter.
The zonal daily-averaged fluxes of NO cooling radiation in several latitude bins are shown in Figure $1 \mathrm{~g}$ for the northern hemisphere and in Figure $1 \mathrm{~h}$ for the southern hemisphere. SABER was in the northern hemisphere viewing mode during 
the event time interval and high southern latitudes were not observed. The highest cooling zonal flux of $\sim 0.45 \mathrm{~mW} / \mathrm{m}^{2}$ is observed in the latitude bin centered around $71.5^{\circ}$ (encompasses the auroral latitude range from $66^{\circ}$ to $77^{\circ}$ ), followed by lesser intensities in $83.5^{\circ}$ and $60.5^{\circ}$-centered bins (nearly overlapping curves). (We use geographic latitude in the paper unless a use of magnetic latitude is explicitly stated.) The lowest levels of NO cooling radiation are observed at low to middle latitudes. The highest flux in the southern hemisphere is observed in the $-60.5^{\circ}$ and $-49.5^{\circ}$ latitude bins. Note the increased cooling radiation in sub-auroral to middle southern latitudes (from $-55^{\circ}$ to $-44^{\circ}$ ) for this event which is not observed for the same latitude range in the northern hemisphere. NO cooling increases at the beginning of the storm and stays nearly constant through the beginning of the recovery phase. Cooling flux slowly decreases by the end of the storm recovery.

Figure 1i shows daily global cooling powers for $\mathrm{NO}$ and $\mathrm{CO}_{2}$. Cooling power of NO shows a stronger response to the storm than $\mathrm{CO}_{2}$ cooling power. Mlynczak et al. (2010a) in their study of multiyear trends in thermospheric cooling indicated that the $\mathrm{CO}_{2}$ cooling is less sensitive than NO cooling to the solar cycle due to the fact that the $\mathrm{CO}_{2}$ emissions originate in the lower (more dense) atmosphere and they are less dependent on storm-related temperature changes. The abundance of $\mathrm{CO}_{2}$ molecules is unlikely to change during geomagnetic activity (Mlynczak et al. 2008). On the other hand, $\mathrm{CO}_{2}$ is the dominant cooling mechanism of the lower thermosphere up to $140 \mathrm{~km}$ in altitude.

Figure $1 \mathrm{j}$ shows the mean HPe summarized from both hemispheres (black dotted line in the middle) and the standard deviation (bounding red lines). HPe starts to increase at the onset of the CIR and the "negative bay" in SYM-H (panels b and $\mathrm{k}$ ) and stays elevated throughout the storm recovery phase. The largest peak in HPe occurs during the HSS storm main phase and corresponds to the largest peaks in the epsilon parameter, Universal function, Kan-Lee field, Joule heating, $\mathrm{AE}$, and a minimum in SYM-H. Note the multiple peaks in HPe which approximately correspond to increases in AE index and are possibly related to electron precipitation during the HILDCAA interval.

\subsection{HSS sub-interval of 25 April-2 May, 2011}

Figure 2 shows interplanetary parameters for the event, solar wind speed (a) and southward component of the interplanetary magnetic field (b). Vertical lines approximately denote times during: the storm main phase ( 20:00 UT on 29 April or 119 DOY, 01:00 UT and 12:00 UT on 30 April or 120 DOY) and the recovery phase ( 23:00 on 1 May or 121 DOY), to be discussed later. The solar wind speed starts to increase to $\sim 700 \mathrm{~km} / \mathrm{s}$ at the end of April 29 (119 DOY). IMF $B_{x}$ changes from negative to positive and IMF $B_{y}$ from positive to negative on 119 DOY which indicates a presence of the HCS occurring well ahead of the HSS and CIR (not shown here to save space). $B_{z}$ in the CIR has smaller amplitude variations for this event than for the previous event. The HSS interval (starting at the end of DOY 119) is associated with gradual ring current development as seen in SYM-H variation (panel k). Interestingly, the peak SYM-H intensity occurs during the HSS proper, unlike the previous case when SYM-H peaks during a CIR event. Variation of the AE index (panel 1) confirms a HILDCAA event with a duration of $~ 58.9 \mathrm{~h}$, from 1804 UT of 30 April to 0456 UT of 3 May. It is characterized by $\mathrm{AE}$ peak and average intensities of $\sim 1407 \mathrm{nT}$ and $\sim 475 \mathrm{nT}$, respectively. The HILDCAA interval is well correlated with high-frequency IMF $B_{z}$ fluctuations embedded in the HSS proper.

The largest peaks in the epsilon parameter, Universal function, Kan-Lee electric field, and Joule heating (panels c-f), and in HPe and AE (panels $\mathrm{j}$ and 1) occur during a two-step decrease in SYM-H (panel k). The epsilon parameter increases abruptly to almost $3000 \mathrm{GW}$ during the main phase, but it is generally lower than for the previous event. It shows smaller peaks corresponding to two consecutive decreases in the SYM-H index. The Universal function (panel d) and Kan-Lee electric field (panel e) reach peaks at the storm onset. More efficient coupling during the HSS phase is reflected in the Universal function (panel d) and Kan-Lee electric field (panel e), which show secondary peaks and elevated values throughout the recovery phase from 30 DOY through 33 DOY. Both Joule heating and Kan-Lee electric field (panels $f$ and e) show multiple peaks, which are sometimes higher than for the previous event and some of which correspond to peaks in HPe (panel j). Joule heating stays increased into the storm recovery phase (126 DOY). In general, peaks in Joule heating correspond in time to peaks in $\mathrm{AE}$ index, but with different intensities.

Verkhoglyadova et al. (2015) also analyzed the 2011 HSS event. They pointed out that larger geomagnetic activity occurred during the HSS (at the solar wind speed maximum on 122 DOY) than during the CIR phase. This phenomenon is captured by peaks in NO cooling radiation in the southern hemisphere at $\sim 0.76 \mathrm{~mW} / \mathrm{m}^{2}$ hemisphere (panel $\mathrm{h}$ ) and in the northern hemisphere at $\sim 0.5 \mathrm{~mW} / \mathrm{m}^{2}$ (panel g, limited latitudinal coverage). SABER was in the southern viewing mode and latitudes above $\sim 55^{\circ}$ in the northern hemisphere were not observed. Note a two-peak structure, corresponding to an increased cooling at the onset of the main phase during the CIR and in the main phase during the HSS. The second peak coincides in time (the same day) with a secondary peak in the coupling functions, i.e., an enhancement in solar wind - magnetosphere coupling. It is unusual that the largest cooling radiation comes from the $\sim-83.5^{\circ}$ latitude bin $\left(\sim-70^{\circ}\right.$ magnetic latitude), followed by flux from the $\sim-71.5^{\circ}\left(\sim-60^{\circ}\right.$ magnetic latitude) auroral bin. There are much lower fluxes at lower latitudes from the southern hemisphere (compare red and green overlapping curves with magenta and blue curves). In the northern hemisphere the largest cooling flux comes from around $\sim 50^{\circ}$ latitude. Figure $2 \mathrm{i}$ shows a multiday increase in NO daily global cooling power and almost no change in $\mathrm{CO}_{2}$ cooling power. $\mathrm{NO}$ power for this event is higher than for the 2007 event.

\section{Modeling of energy transport with GITM}

We apply GITM to quantify energy inputs and sinks in the IT system in the altitude range between $100 \mathrm{~km}$ and $600 \mathrm{~km}$ as a function of latitude and local time throughout the HSS-caused storms. Modeling allows us to create global distributions of Joule heating, auroral heating, and different cooling channels throughout the events. We focus on driving the system "from above" and utilize empirical neutral atmosphere models for the lower boundary conditions at $100 \mathrm{~km}$. Thus, we do not consider an important aspect of a self-consistent interaction of the E-region dynamo with tides (Liu et al. 2010) which is the subject of a separate study. We would like to note that "driving IT from below" in several instances can cause effects 


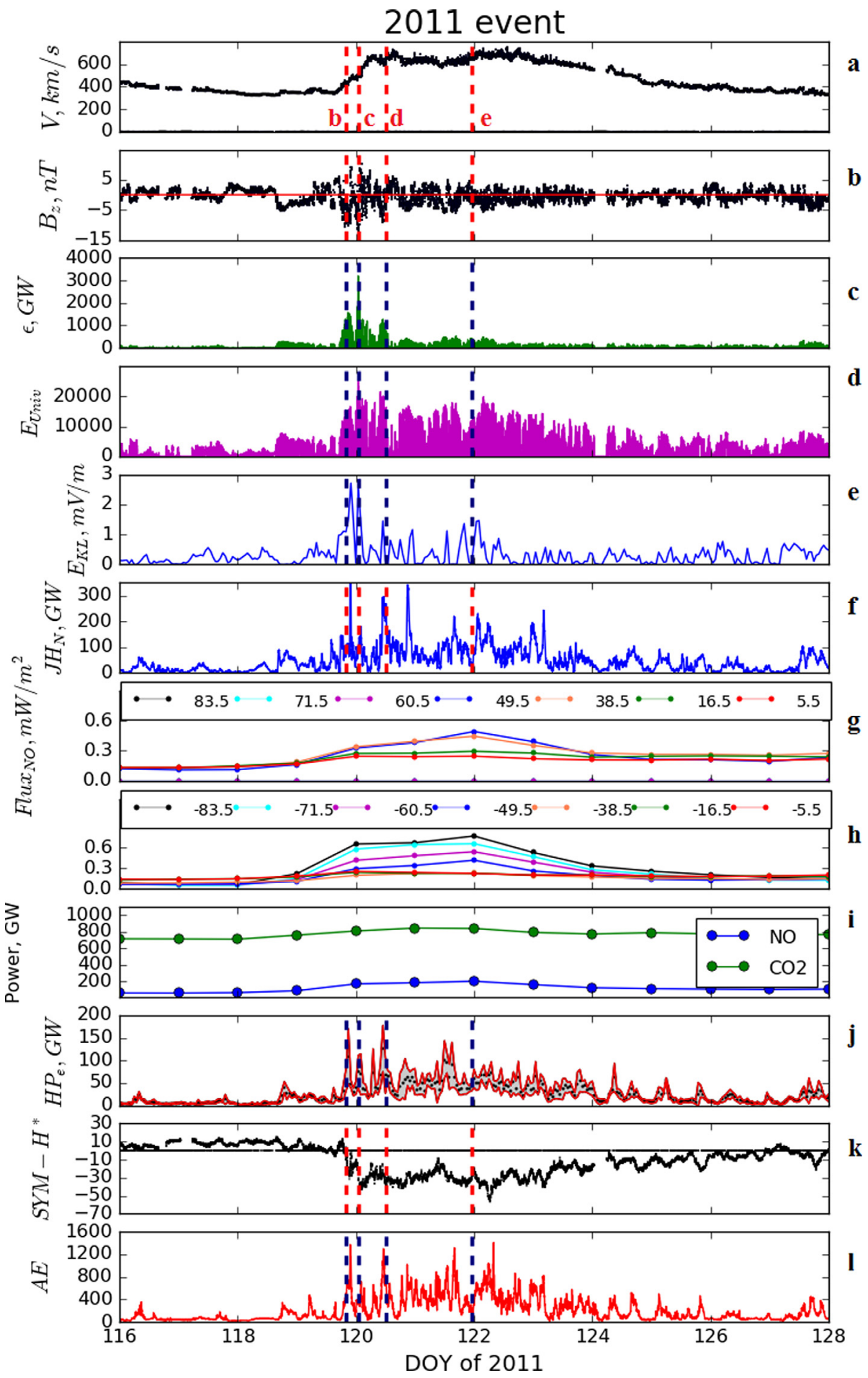

Fig. 2. The same as in Figure 1 but for the 2011 CIR-HSS event. Vertical dashed lines indicate sample times during: the storm main phase ( 20:00 UT on 29 April or 119 DOY, snapshot b; 01:00 UT and 12:00 UT on 30 April or 120 DOY, snapshots c and d) and recovery phase ( 23:00 on 1 May or 121 DOY, snapshot e). Snapshot numbering is shown in panel a. See text for details.

comparable to external driving, e.g. (Hagan et al. 2015) and requires detailed analysis for individual events (see also Sect. 4).

We utilize a "forecasting mode" in our modeling approach which means that all major inputs are forecastable (Mannucci et al. 2015) and assume the availability of a solar wind forecast with a lead time of 1-2 days. The model run does not use ad hoc adjustments to specific events, i.e., no specific "tuning" was applied. One of the study goals is to understand if we can capture the main physics of the energy budget in a forecast and to compare with observation-based estimates. We acknowledge that the observational side is represented by approximate and globally averaged energy estimates, and address this issue in Section 4 in some detail. Although the height-integrated modeled quantities are calculated only within the model altitude range, i.e., from $100 \mathrm{~km}$ to $600 \mathrm{~km}$, we expect that the main energy release into IT during moderate storms takes place within these boundaries (Huang et al. 2012b). 
In this section we investigate whether modeling can successfully quantify the energy budget and show possible shortcomings of modeling to be improved upon. We seek to address: (a) how do modeled auroral heating, Joule heating, and NO cooling correspond to a geomagnetic storm timeline? (b) Are substantial changes (increases or decreases) in these parameters throughout the events physical? (c) Do peak values agree with the empirical estimates? (d) How well does the global energy budget agree with physical concepts of a HSS storm and coupling functions? (e) What are differences and similarities between the two storms?

\subsection{Brief description of GITM and specifications of the model runs}

At the core of GITM are the continuity, momentum, and energy equations for neutrals and ions (Ridley et al. 2006). GITM includes comprehensively the physical processes in the ionosphere and thermosphere, such as solar EUV heating, auroral particle precipitation, chemical reactions, ion-neutral and neutral-neutral collisions, viscosity, heat conduction, radiative cooling, and high-latitude electric field driving. The main energy sinks in the model are conduction, cooling emissions in atomic oxygen (O), $\mathrm{NO}$, and $\mathrm{CO}_{2}$ lines (Ridley et al. 2006). The model allows for non-hydrostatic solutions, i.e., it describes dynamic processes outside of hydrostatic equilibrium. GITM computes on geographic longitude, latitude, and altitude grids. For a global simulation, the vertical domain covers altitudes from $100 \mathrm{~km}$ to around $600 \mathrm{~km}$ in altitude.

The initial atmospheric state and lower boundary conditions are taken from the empirical atmospheric models of MSIS (Mass Spectrometer and Incoherent Scatter Radar model: Hedin 1991) and HWM (Horizontal Wind Model: Drob et al. 2008). General inputs to the GITM are the solar wind and IMF conditions, solar irradiance, and hemispheric power indices. Daily F10.7 flux is taken from the Space Physics Interactive Data Resource (http://spidr.ngdc.noaa.gov/spidr/home.do). The Flare Irradiance Spectral Model (FISM; Chamberlin et al. 2007) provides empirical solar irradiance spectra (http:// lasp.colorado.edu/lisird/fism/). We perform global GITM runs with different sets of auroral precipitation and high-latitude electric field patterns. Our primary runs use the Weimer05 model (Weimer 2005) with 1-minute solar wind inputs to represent the high-latitude electric field. For comparison of the total energy budget we also use the Assimilative Mapping of Ionospheric Electrodynamics (AMIE) formulation (Richmond \& Kamide 1988; Ridley \& Kihn 2004) to provide the highlatitude electric potential and field-aligned currents to the GITM. AMIE uses ground magnetometer data (for our simulations, the data are downloaded from the SuperMAG website: http://supermag.jhuapl.edu/).

We utilize the Oval Variation, Assessment, Tracking, Intensity, and Online Nowcasting (Ovation) Prime model by Newell et al. (2009) as the auroral precipitation module in GITM. The model predicts intensity for four types of aurora at different locations and estimates the equatorward auroral boundary. Corresponding model runs are referred to as the OP (Ovation Prime) runs.

Alternatively, we use the more traditional approach based on 9 NOAA hemispheric power index (HPI) patterns (see Sect. 1). Based on detailed analysis of DMSP measurements, Hardy et al. (2008) showed that about $90 \%$ of the total auroral energy flux (or $\mathrm{HPt}$ ) is carried by electrons with energies between $660 \mathrm{eV}$ and $30 \mathrm{keV}$. GITM uses HPt from NOAA
POES satellites only, which are revised to agree with NOAA$12 \mathrm{HPt}$ estimates after 1990, and are thus approximately 1.75 times larger than the inter-calibrated HPt estimates of Emery et al. (2008) from DMSP and NOAA satellites. HPt is an integral quantity calculated using the estimates of electron fluxes along a NOAA POES satellite orbital pass referenced to the HPt NOAA statistical global energy flux patterns from FullerRowell \& Evans (1987) for different levels of geomagnetic activity ordered by HPI. This model is hereafter denoted as NOAA HP and is used with both Weimer05 and AMIE in GITM.

To summarize, we use three different types of model runs throughout the paper with the following modules describing solar irradiance, auroral particle precipitation, and high-latitude electrodynamics: (1) Weimer05-driven combined with NOAA HP and FISM (referred to as NOAA HP or HP-driven); (2) AMIE-driven combined with NOAA HP and FISM (referred to as AMIE-driven); (3) Weimer05-driven combined with Ovation Prime (OP) and F10.7 (referred to as OP-driven). UV heating tends to stay fairly constant throughout the geomagnetic storms (Fig. 3 shows a small difference in the EUV heating powers across the models). Thus, we believe these differences in EUV input are not important for the current study which is focused on time-dependent storm responses.

\subsection{Modeling results for the 2007 event}

For a detailed study we show several hourly estimates of the IT energy budget (GITM output is provided every hour unless stated otherwise) corresponding to distinct phases of the HSS event shown approximately by the vertical lines in Figure 1 and indicated in red on panel a, i.e., pre-event background, the storm main phase, and recovery phase, approximately corresponding to 24 January 2007 at 00 UT (a), 29 January 2007 at 07 UT (b), 29 January 2007 at 14 UT (c), and 31 January 2007 at 05 UT (e). We will also show results for another snapshot during the main phase at $\sim 15: 00$ UT on 29 January (d) for comparison. Note that the event "background" coincides with the end of a preceding HSS, but corresponding geomagnetic activity and IT responses are very low (Fig. 1). Figure 3 shows how the IT energy budget is partitioned between the main energy inputs (heating) and sinks (cooling) in GITM and averaged over the globe. The left panels show model estimates for the partial energy power densities at $125 \mathrm{~km}$ altitude (in $\mathrm{W} / \mathrm{m}^{3}$ ), and the right panels show power densities integrated from $100 \mathrm{~km}$ to $600 \mathrm{~km}$ altitude (in $\mathrm{W} / \mathrm{m}^{2}$ ). Total heating and cooling powers calculated with different external driving of the GITM are shown on the panels.

Figure 3a shows globally averaged energy partitioning during the relatively quiet pre-event background (snapshot a, see Fig. 1a) at the reference altitude of $125 \mathrm{~km}$. All energy inputs and sinks are low in value and about equal among themselves. Photoelectron heating, auroral heating, and conduction cooling are negligible during the quiet-time. Figure $3 b$ shows results at the storm onset and before the CIR encounter (snapshot b). Energy input into the IT at $125 \mathrm{~km}$ is split almost equally between Joule heating and auroral heating with slightly higher Joule heating compared to the auroral heating driven by OP, following by the solar EUV (sunspot number is very low at $\sim 25$ and this energy input stays nearly the same throughout the storm event) and chemical heating. The major cooling factors are $\mathrm{O}$ and $\mathrm{NO}$ cooling emissions.

At the main phase onset (snapshot c), the main energy input at $125 \mathrm{~km}$ comes through Joule heating, while other 
Global Heating Budget at $125 \mathrm{~km}$ Altitude

(a)

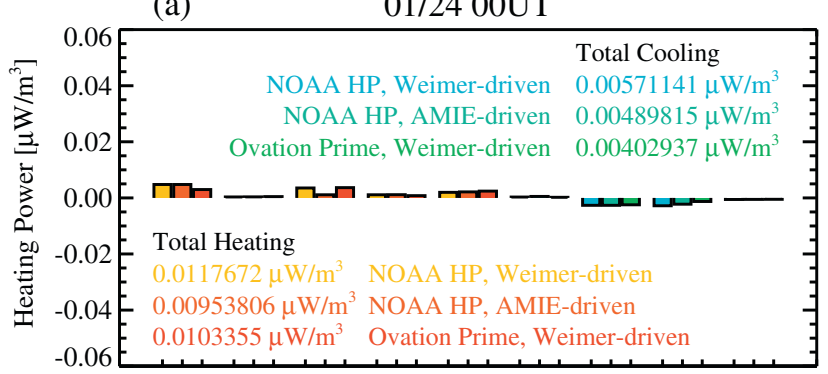

(b)

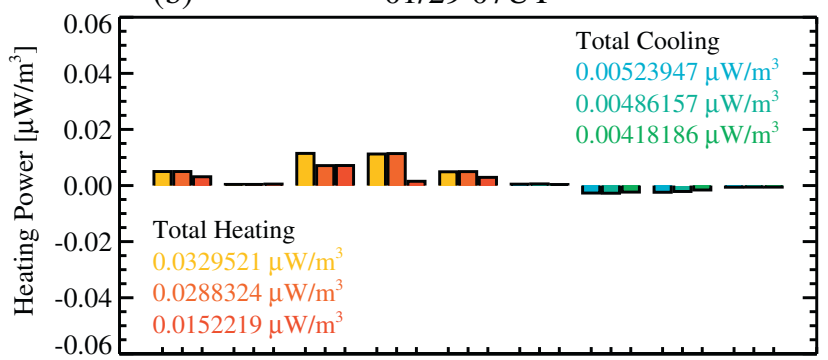

(c)

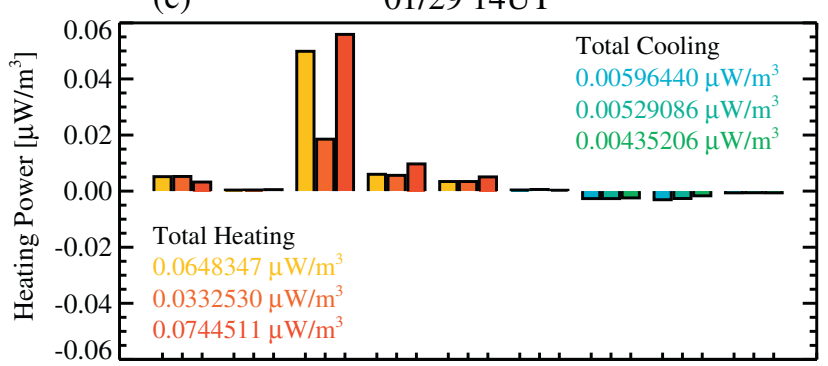

(d)

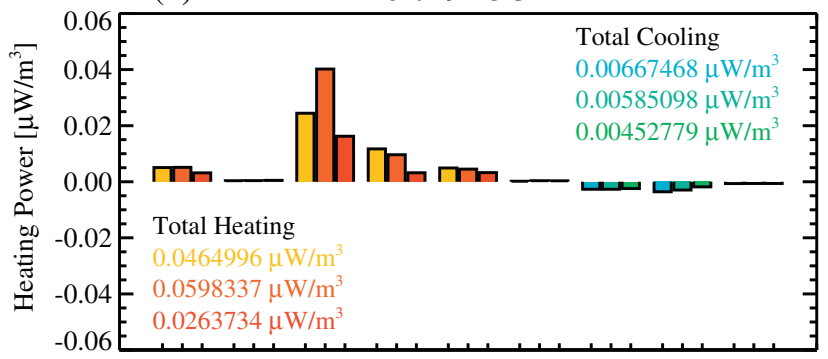

(e)

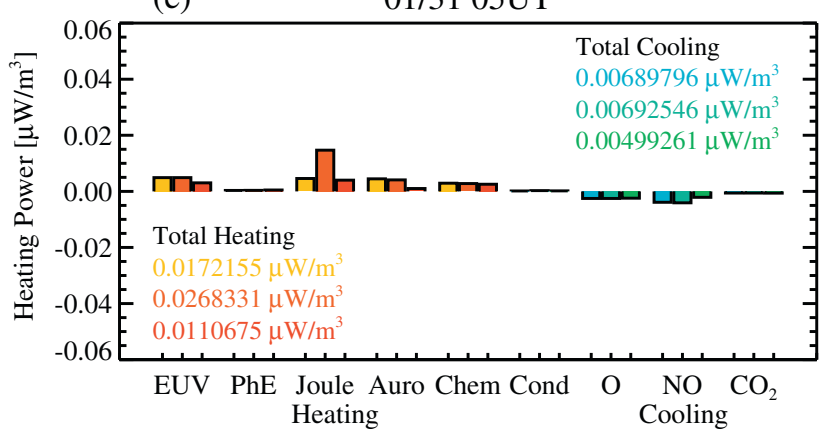

Global Heating Budget, Height-integrated
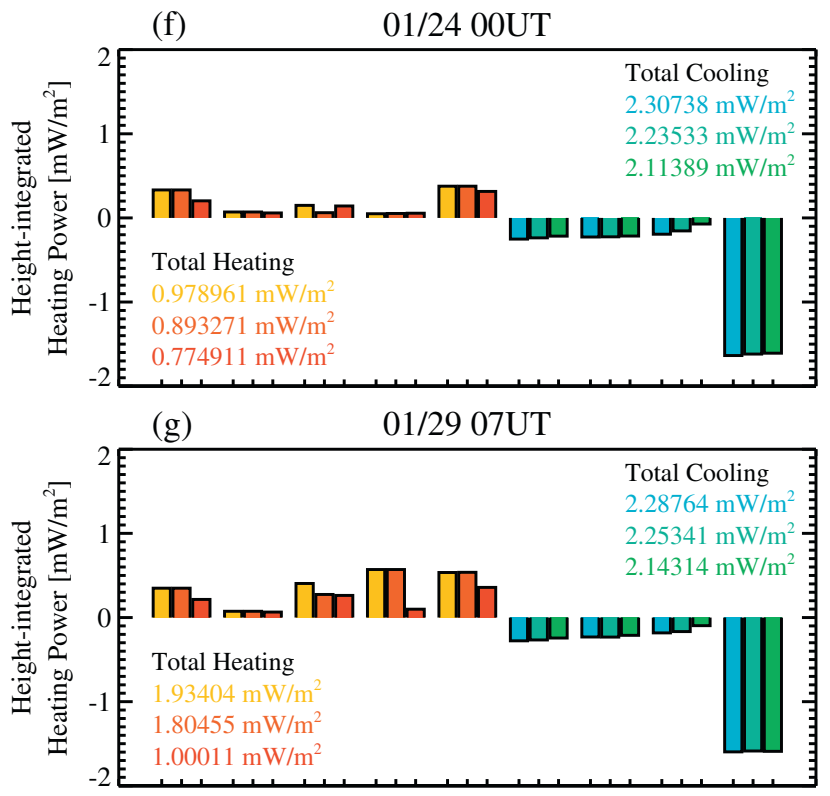

(h)

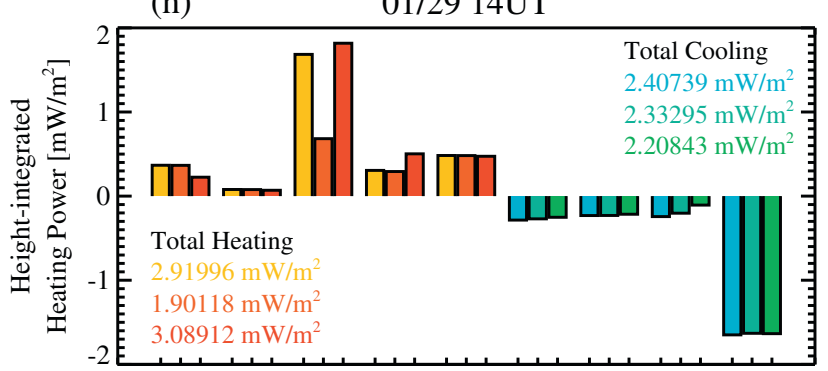

(i)
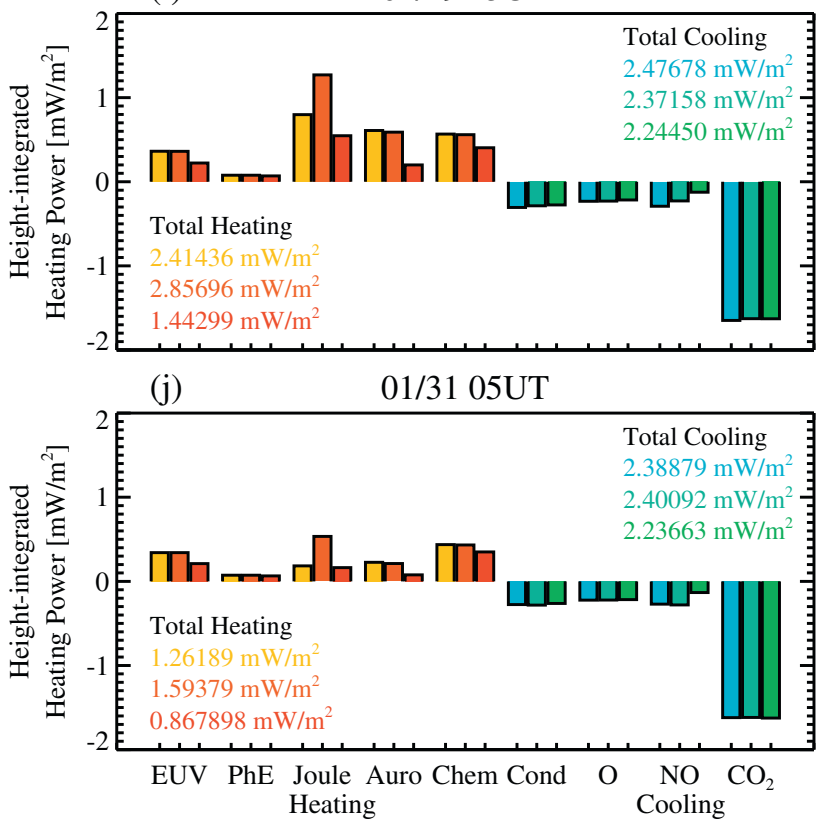

Fig. 3. Globally averaged heating budget of the IT from GITM modeling at $125 \mathrm{~km}$ altitude (a-e) and height-integrated (f-j) on: 24 Jan 2007 at 00 UT, 29 Jan 2007 at 07 UT, 29 Jan 2007 at 14 UT, 29 Jan 2007 at 15 UT, and 31 Jan 2007 at 05 UT (from top to bottom). The total heating and cooling estimated for different energy channels are shown on the panels. Total heating and cooling summed over all energy channels are indicated on the panels. Estimates are made for three different GITM runs (shown by different colors). See text for details.

inputs do not change much (Fig. 3c). The largest heating is shown in the NOAA HP (and Weimer05) and OP runs, and the smallest is in the AMIE-driven run. As the storm progresses (Fig. 3d and snapshot d), auroral heating input also increases. Note that panels (c) and (d) show energy partitioning at times $1 \mathrm{~h}$ apart. The AMIE-driven run shows heating twice 


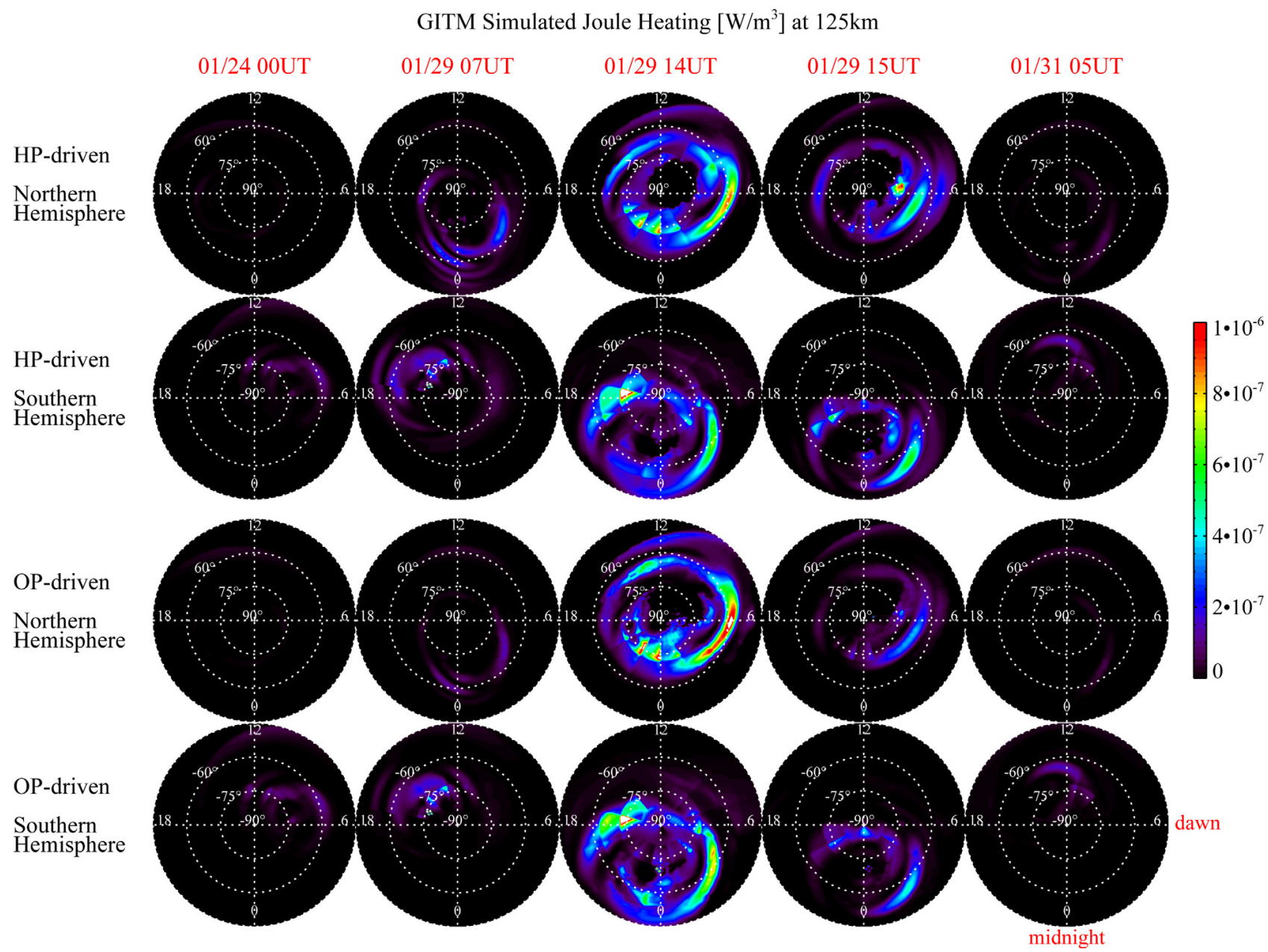

Fig. 4. Joule heating density at $125 \mathrm{~km}$ altitude from the GITM plotted for the northern hemisphere with the HP driving, for the southern hemisphere with the HP driving, for the northern hemisphere with the OP driving, and for the southern hemisphere with the OP driving (rows from top to bottom). Columns correspond to the times of snapshots shown in Figure 3.

as large as the OP run at the later time. Thus, by comparing Figures $3 \mathrm{c}$ and $3 \mathrm{~d}$ we conclude that Joule heating is highly variable at this timescale. Joule heating estimates during the storm main phase differ substantially between different models. There is a difference in timing while driving with different particle precipitation model and polar cap potential patterns.

Energy inputs start to recover to a pre-event background at the storm recovery phase (Fig. 3e and snapshot e). Energy sinks stay about the same throughout the event. NO cooling increases after the main phase (not shown here). If we compare total heating and total cooling at $125 \mathrm{~km}$ during the storm main phase (panels c and d, text), the heating is about an order of magnitude higher than cooling. This could mean that even though energy is deposited at $125 \mathrm{~km}$ it is released from the IT at different altitudes to maintain the energy balance.

The globally averaged height-integrated energy budget is presented in the right panels in Figure 3. Energy input into the IT system is balanced by $\mathrm{CO}_{2}$ cooling (mostly at the lower boundary of GITM at $\sim 100 \mathrm{~km}$ ), which stays about constant throughout the event (compare with Fig. 1i). Joule heating and auroral heating increase during the HSS storm (compare panels $h$ and $i$ with panels $f$ and $j$ ). Outputs of different model runs are markedly different in magnitude and timing of the increases. Detailed analysis of the energy budget timeline shows that height-integrated NO cooling and conduction also increase during the storm. Corresponding cooling intensities show a strong altitude dependence.

The inputs and sinks do not balance (Fig. 3, panels $\mathrm{f}-\mathrm{j}$, text), with the heating power increasing $\sim 3$ to 4 times during the storm relative to the pre-event values and cooling staying nearly constant. Height-integrated cooling in our modeling is dominated by $\mathrm{CO}_{2}$ cooling which does not show a noticeable dependence on the storm phases. In their analysis of atmospheric dynamics and chemistry Mlynczak et al. (2008) suggested that $\mathrm{CO}_{2}$ emission is much less sensitive to geomagnetic activity than NO emission.

Figure 4 shows Joule heating at the characteristic altitude of $125 \mathrm{~km}$ for the same snapshots as in Figure 3. The two model runs include: (1) NOAA HP and Weimer05 and (2) OP and Weimer05. Note that solar EUV input is also different, with the FISM used with NOAA HP and F10.7 index used with OP. We use the first combination for the GITM modeling input because it is the most typical setting and has been well validated. The second model run setting is to configure GITM in a forecast mode, i.e., all inputs to GITM are forecastable.

Each panel shows modeling results for driving highlatitude electrodynamics with the Weimer05 model and a particle precipitation pattern defined either by NOAA HP (two top rows) or Ovation Prime (two bottom rows). The top plots represent the Northern hemisphere $(\mathrm{NH})$ and bottom plots 


\section{GITM Simulated Height-integrated Joule Heating $\left[\mathrm{W} / \mathrm{m}^{2}\right]$}

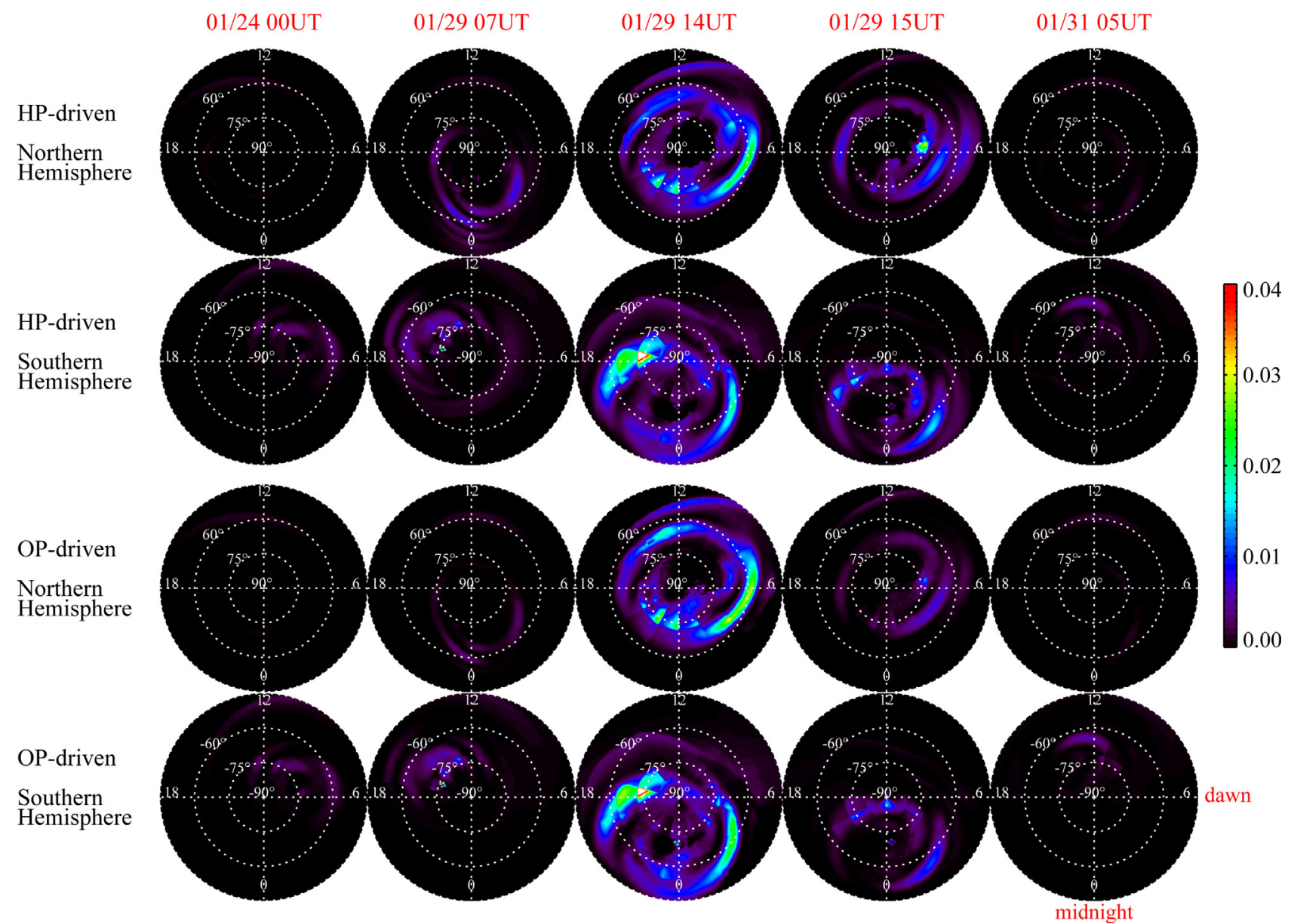

Fig. 5. Height-integrated Joule heating density from the GITM model plotted for the northern hemisphere with the HP driving, for the southern hemisphere with the HP driving, for the northern hemisphere with the OP driving, and for the southern hemisphere with the OP driving (rows from top to bottom). Columns correspond to the times of snapshots shown in Figure 3.

represent the Southern hemisphere $(\mathrm{SH})$ in each pair of runs. Note that the Northern hemisphere is viewed as though one is looking toward the Earth and the Southern hemisphere is viewed as though one is looking through the Earth. Maps are made in geographic coordinates and local times. For comparison purposes all maps are made on the same scale. At the event onset, there is very little Joule heating in the post-midnight sector, which is stronger in the case of driving by the NOAA HP (two left columns). Note the inter-hemispheric symmetry in Joule heating distribution. During the storm main phase, both HP- and OP-driven modeling show the Joule heating intensifications in the midnight and morning sectors in the NH (the middle column). In the SH the intensifications are located in the dusk and post-midnight sectors. We start to observe an asymmetry between the hemispheres. Timing of the intensifications coincides with peaks in the coupling functions (Figs. 1c-1e). Runs with the Weimer05 model and different models of particle precipitation (HP and OP) give very similar Joule heating patterns, but different magnitudes. Global distribution of Joule heating is dynamical on an hourly scale (next column). Joule heating remains intensified in the dawn sector in the $\mathrm{NH}$ and in the post-midnight sector in the $\mathrm{SH}$. It becomes small during the recovery phase (right column).

Figure 5 shows height-integrated Joule heating at times corresponding to the panels in Figure 4. The global distribution is almost identical to the distribution at $125 \mathrm{~km}$. This could imply that the main portion of Joule heating is mostly a contribution from $\sim 125 \mathrm{~km}$ since the patterns are about the same (in agreement with Huang et al. 2012a).

Figure 6 presents height-integrated NO cooling for the same time snapshots. There are notable differences in modeling results depending on the particle precipitation model, i.e., driven by NOAA HP or Ovation Prime. The former produces NO cooling emission almost two times stronger at selected times, which spreads down to $15^{\circ}$ latitude. Both inputs show a similar global pattern of $\mathrm{NO}$ emission. Modeling shows consistently larger cooling in the summer SH for this event. The cooling emission is quite large even for the pre-event background (left columns). The peak NO emission is in low latitudes at dusk. It moves to the noon sector for the main phase. Comparison of the panels for 14 UT and 15 UT shows that cooling features persist for at least one hour. However, a lack of cooling emission in high latitudes is markedly different from observations (see Figs. 1g and $1 \mathrm{~h}$ ), where the winter $\mathrm{NH}$ high latitudes show the largest NO cooling emission fluxes from SABER. NO cooling maps at $125 \mathrm{~km}$ altitude show a similar pattern as the height-integrated NO cooling (not shown here).

Figure 7 presents height-integrated auroral heating for the same snapshots as in Figure 4. Pronounced auroral heating is 


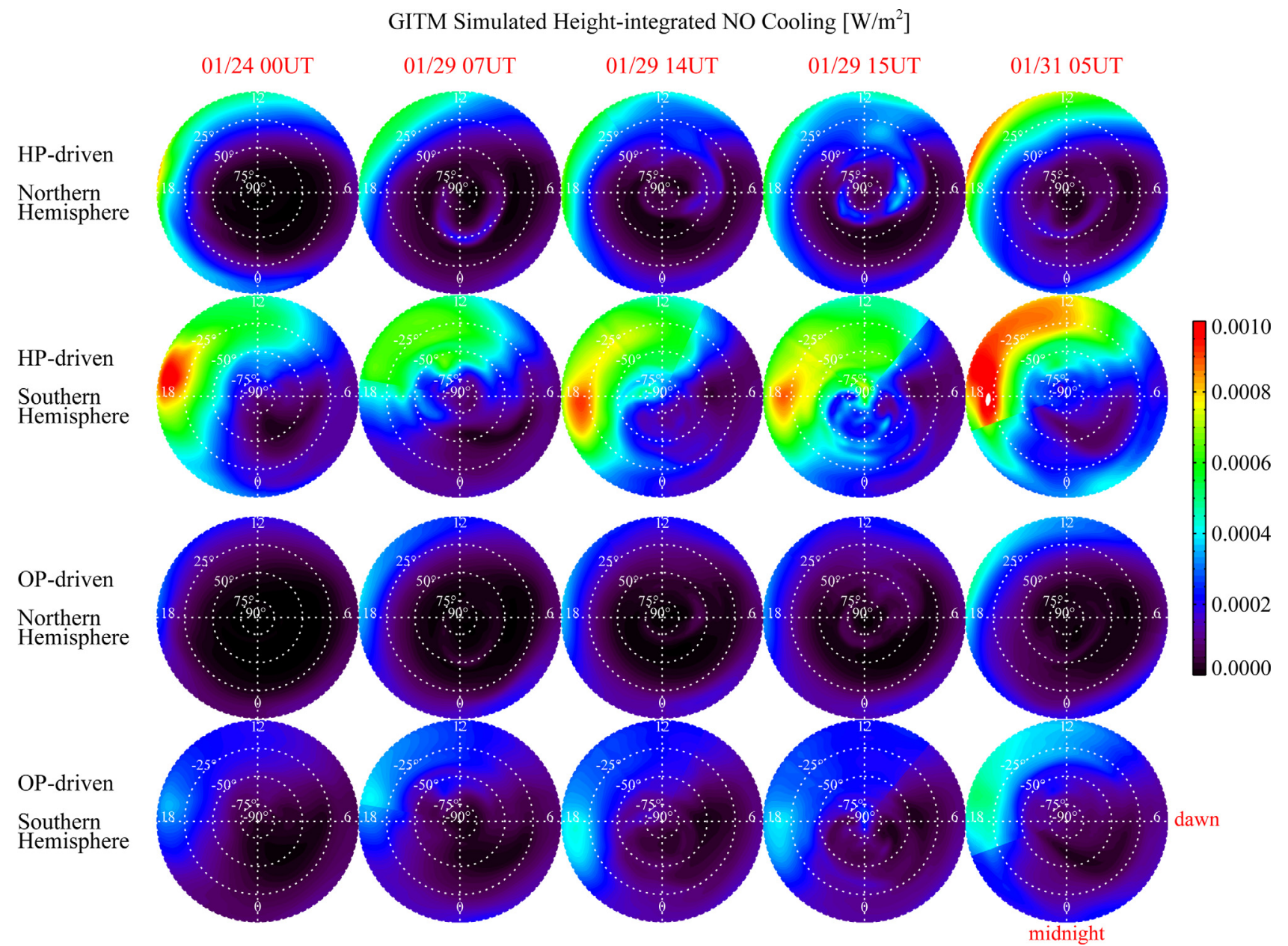

Fig. 6. Height-integrated NO cooling emission power density from the GITM model plotted for the northern hemisphere with the HP driving, for the southern hemisphere with the HP driving, for the northern hemisphere with the OP driving, and for the southern hemisphere with the OP driving (rows from top to bottom). Columns correspond to the times of snapshots shown in Figure 3.

driven by NOAA HP during the initial phase (top rows). No heating is noted for the case of OP driving (bottom plots). At the storm onset, auroral heating driven by NOAA HP diminishes but auroral heating driven by Ovation Prime increases. In an hour the HP-driven heating pattern intensifies and the OP-driven pattern diminishes. Note small interhemispheric asymmetry of heating driven by precipitating particles, with the peaks around dawn and pre-midnight local time in the $\mathrm{NH}$ and in the $\mathrm{SH}$ for the main phase (1400 UT and 1500 UT on 29 January). In general, auroral heating driven by different models peaks at different times. Auroral heating substantially decreases during the recovery phase (last column). Auroral heating mapped at $125 \mathrm{~km}$ altitude shows very similar global distributions as the height-integrated quantity, but with finer resolved small-scale spatial features (not shown here).

\subsection{Modeling results for the 2011 event}

For a detailed study we present several hourly snapshots of energy partitioning in the HSS event: (a) a pre-event background ( 12:00 UT on 25 April or 115 DOY), (b) the storm main phase ( 20:00 UT on 29 April or 119 DOY), (c) the storm main phase ( 01:00 UT on 30 April or 120 DOY), (d) $\sim 12: 00$ UT on 30 April or 120 DOY, for comparison of IT dynamics, and (e) recovery phase $(\sim 23: 00$ on 1 May or
121 DOY). Times of snapshots (b) through (e) are shown approximately by the vertical lines in Figure 2 and indicated in panel a. Figure 8 shows how the total globally averaged heating budget is partitioned between the main energy inputs and sinks in GITM. Left panels show model estimates for the partial energy powers at $125 \mathrm{~km}$ altitude, and right panels show quantities integrated from $100 \mathrm{~km}$ to $600 \mathrm{~km}$ altitude. Total heating and cooling powers calculated with different external driving of the GITM are shown on the panels.

Pre-event background (panels a and f) shows low-energy input and cooling by $\mathrm{O}$ and $\mathrm{NO}$ emissions at $125 \mathrm{~km}$, and mostly EUV and chemical heating with dominant $\mathrm{CO}_{2}$ cooling for the height-integrated power. The main phase of the HSS storm is characterized by very intense Joule heating (panels $\mathrm{b}-\mathrm{d}$ for Joule heating at $125 \mathrm{~km}$ and panels $\mathrm{g}-\mathrm{i}$ for the height-integrated Joule heating). Note the estimated peak Joule heating across different models (driven either by Weimer05 or by AMIE) can differ by a factor of 2 or larger. Joule heating for models driven by Weimer05 with different auroral particle precipitation shows smaller differences. Auroral heating is the second largest energy input during geomagnetically disturbed intervals. Comparison of total heating and total cooling at $125 \mathrm{~km}$ during the storm main phase (panels $\mathrm{c}$ and $\mathrm{d}$, text) indicates that the heating is about an order of magnitude higher than cooling, which is also the case for the previous event modeling. 
GITM Simulated Height-integrated Auroral Heating [W/m²]

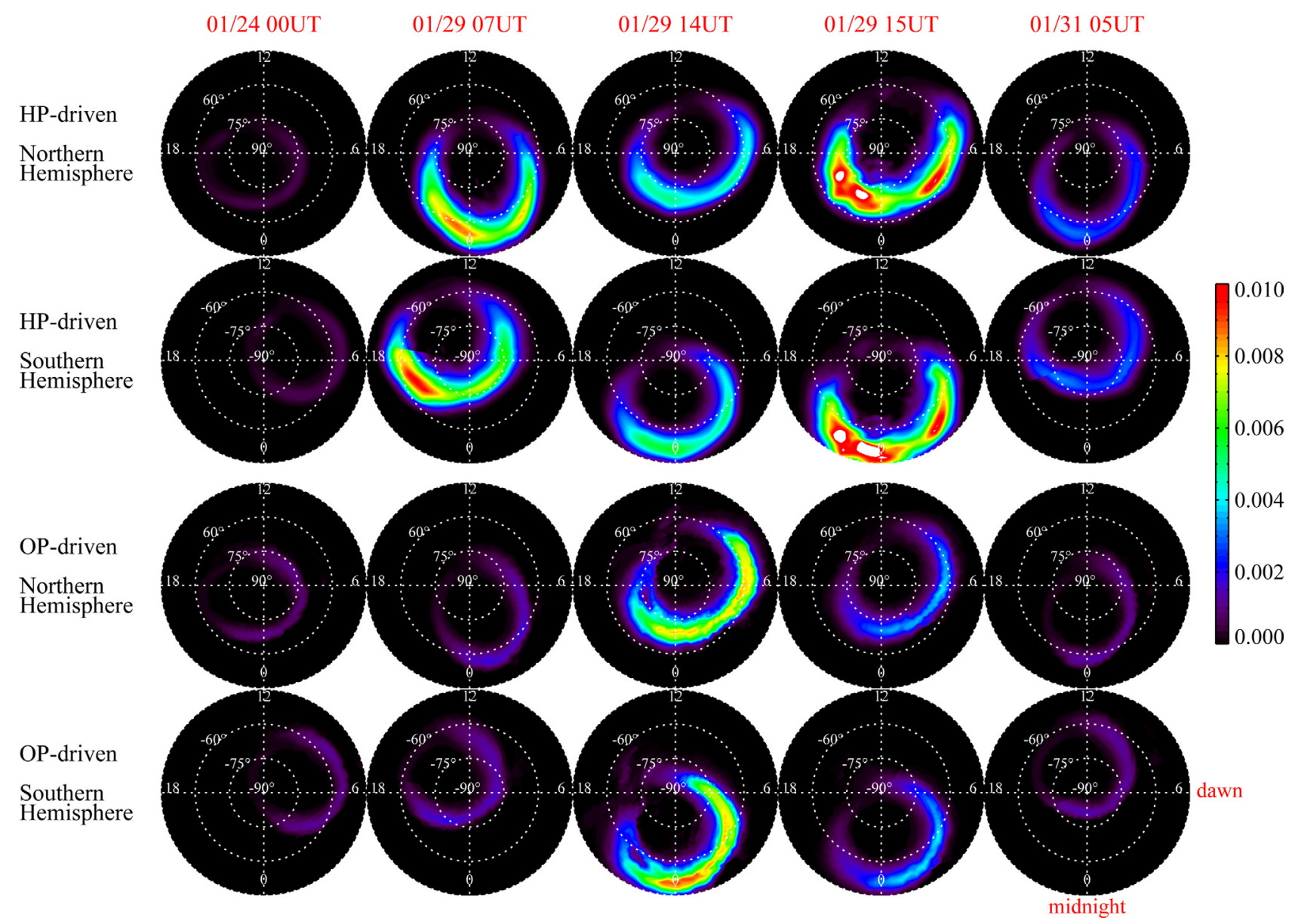

Fig. 7. Height-integrated auroral heating power density from the GITM model plotted for the northern hemisphere with the HP driving, for the southern hemisphere with the HP driving, for the northern hemisphere with the OP driving, and for the southern hemisphere with the OP driving (rows from top to bottom). Columns correspond to the times of snapshots shown in Figure 3.

Height-integrated $\mathrm{CO}_{2}$ cooling is the dominant in absolute value and it originates from $\sim 100 \mathrm{~km}$ altitude. Cooling by conduction is the second largest cooling mechanism among height-integrated quantities. This agrees with the modeled dynamics for the previous event plotted on the same scale in Figure 3. The recovery phase snapshot (panels e and j) shows a recovery toward background values (compare with panels a and $\mathrm{f}$ ). This corresponds in time to a local minimum in coupling functions, Kan-Lee electric field, Joule heating, HPe, and $\mathrm{AE}$ index (see Fig. 2).

The total heating and cooling do not balance (panels $\mathrm{f}-\mathrm{j}$, text), with the heating power increasing 3 to 3.5 times relative to the pre-event values during the main phase of the storm and cooling staying nearly constant. Again as for the previous event, the total cooling in the modeling is dominated by $\mathrm{CO}_{2}$ cooling which does not show a dependence on the storm phases and may not be realistic. Below we focus on the dynamics of height-integrated Joule heating, NO cooling, and auroral heating for the snapshots (b) through (e).

Figure 9 shows GITM simulated height-integrated Joule heating in the same format as in Figure 5. The corresponding pattern at $125 \mathrm{~km}$ is similar to the height-integrated one for the previous event (not shown here). At the event onset and the main phase (see Fig. 2 for the event timing) we observe intensification in Joule heating with clear inter-hemispheric asymmetry in intensity. The most intense heating occurs at 01:00 UT on 30 April (the middle column) which corresponds to the coupling function maxima (Figs. 2c-2e). Driving by OP appears to be stronger (bottom panels) than by NOAA HP, but the Joule heating pattern during the main phase appears to be similar for all inputs throughout the event. Joule heating almost ceases at the recovery phase (snapshot e).

Figure 10 shows the height-integrated NO cooling emission power column density in the same format and for the same snapshots as in Figure 9. Cooling patterns are similar across different particle precipitation models unlike in the previous event. Ovation Prime driving during the main phase of the storm and NOAA HP driving during the recovery phase cause somewhat stronger NO cooling. Stronger cooling is observed in the NH especially at the recovery phase on May 1 . This is in agreement with the major peak in observed NO cooling flux (Fig. 1h). There is hemispheric symmetry in NO emission during the main and recovery phases. Note that cooling is the strongest in middle to low latitudes and is in agreement with modeling results for the previous event (Fig. 6). However, observations show that NO cooling emission flux is dominant in the high-latitude thermosphere (Figs. $2 \mathrm{~g}$ and $2 \mathrm{~h}$ ). Similar to the previous event, the modeling shows the strongest cooling in the summer NH lower latitudes, while the observations show the strongest emission in the winter SH higher latitudes. 
O. Verkhoglyadova et al.: Energy Budget of Ionosphere-Thermosphere During Two CIR-HSS Events

Global Heating Budget at $125 \mathrm{~km}$ Altitude

(a)

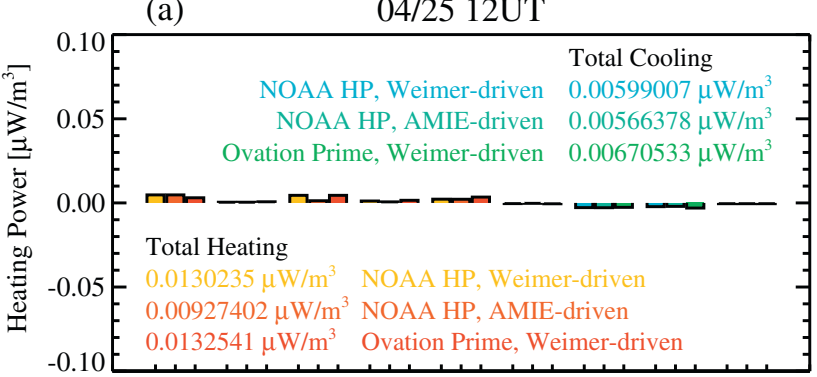

(b)

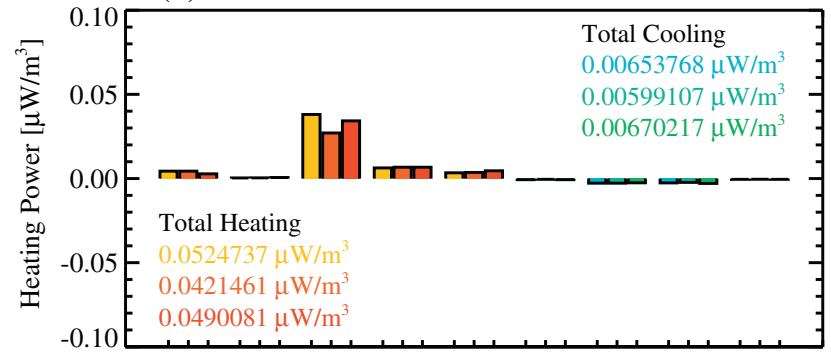

(c)

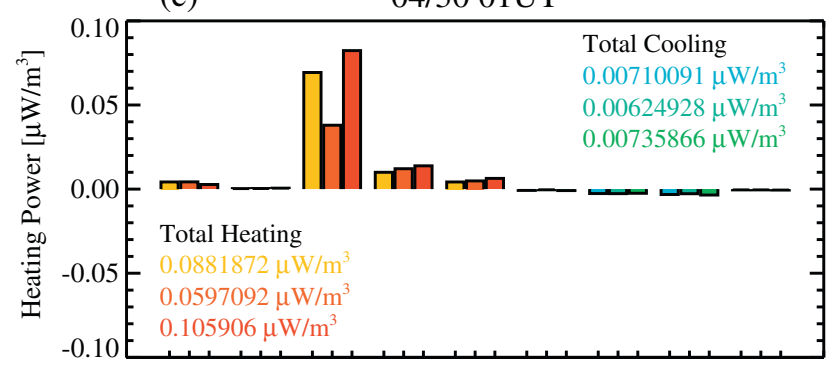

(d)

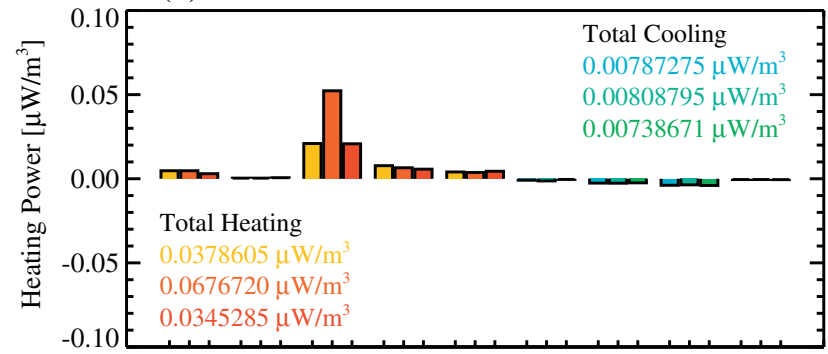

(e)

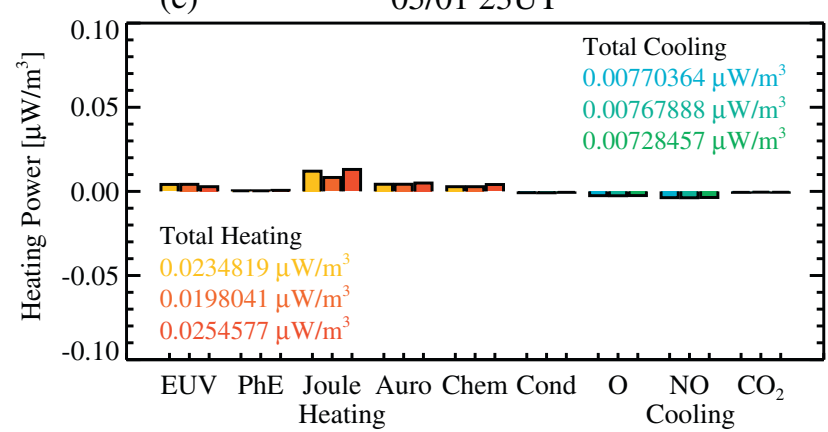

Global Heating Budget, Height-integrated

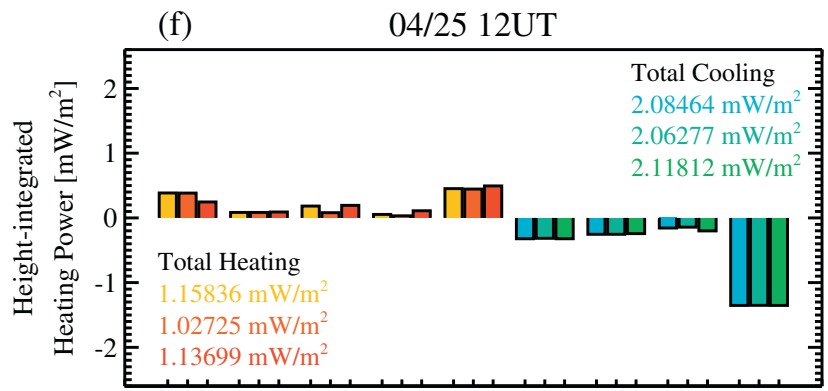

(g)

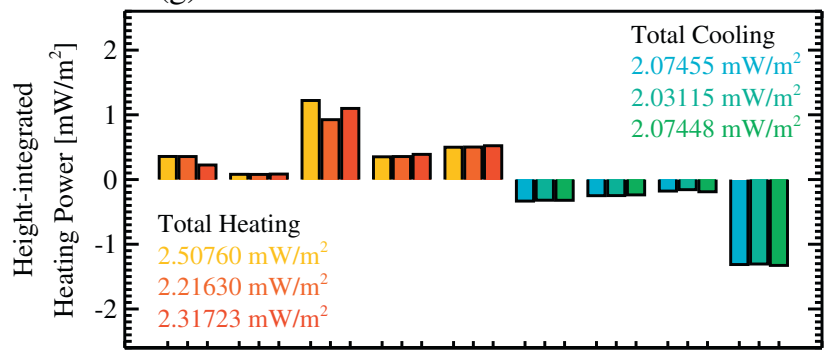

(h)

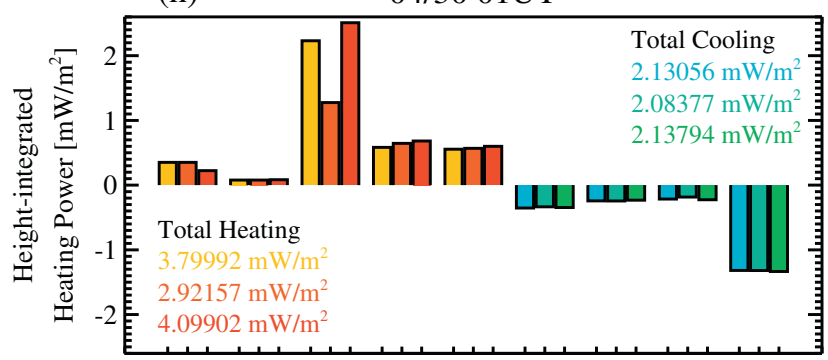

(i)

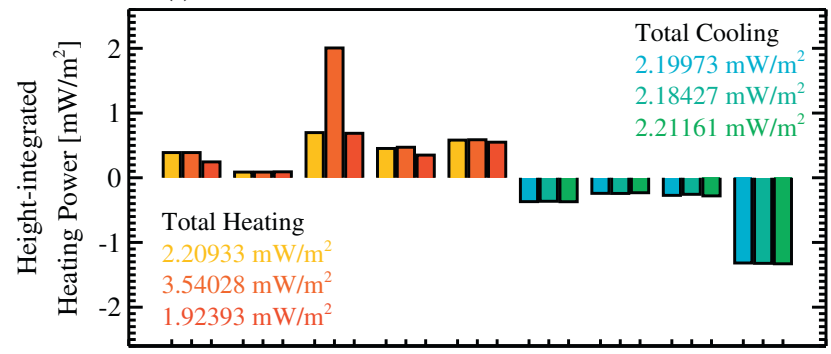

(j) $\quad 05 / 0123 \mathrm{UT}$

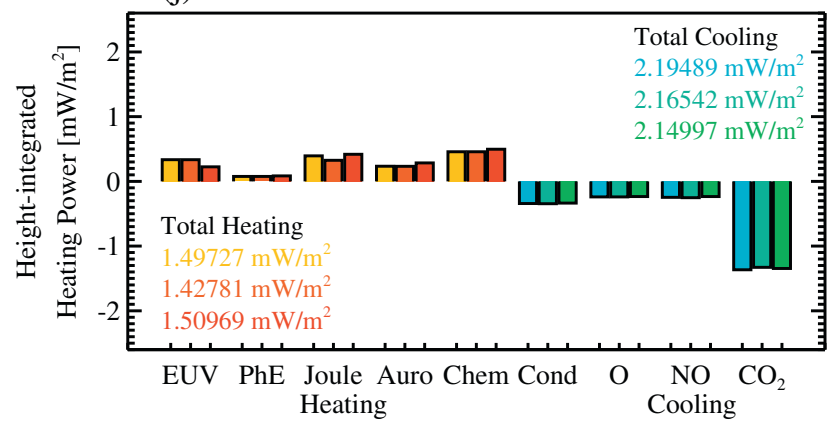

Fig. 8. Globally averaged heating budget of the IT from GITM modeling at $125 \mathrm{~km}$ altitude (a-e) and height-integrated (f-j) on: 25 April 2011 at 12 UT, 29 April 2011 at 20 UT, 30 April 2011 at 01 UT, 30 April 2011 at 12 UT, and 1 May 2011 at 23 UT (from top to bottom). The total heating and cooling estimated for different energy channels are shown on the panels. Total heating and cooling summed over all energy channels are indicated on the panels. Estimates are made for three different GITM runs (shown by different colors). See text for details.

Figure 11 shows height-integrated auroral heating in the same format as in Figure 10. Note the inter-hemispheric asymmetry of heating before the event. During the storm main phase, NOAA HP- and OP-driven auroral heating are close to being symmetric across different hemispheres, with the peaks around dawn and pre-/post-midnight local time. Ovation 


\section{GITM Simulated Height-integrated Joule Heating $\left[\mathrm{W} / \mathrm{m}^{2}\right]$}

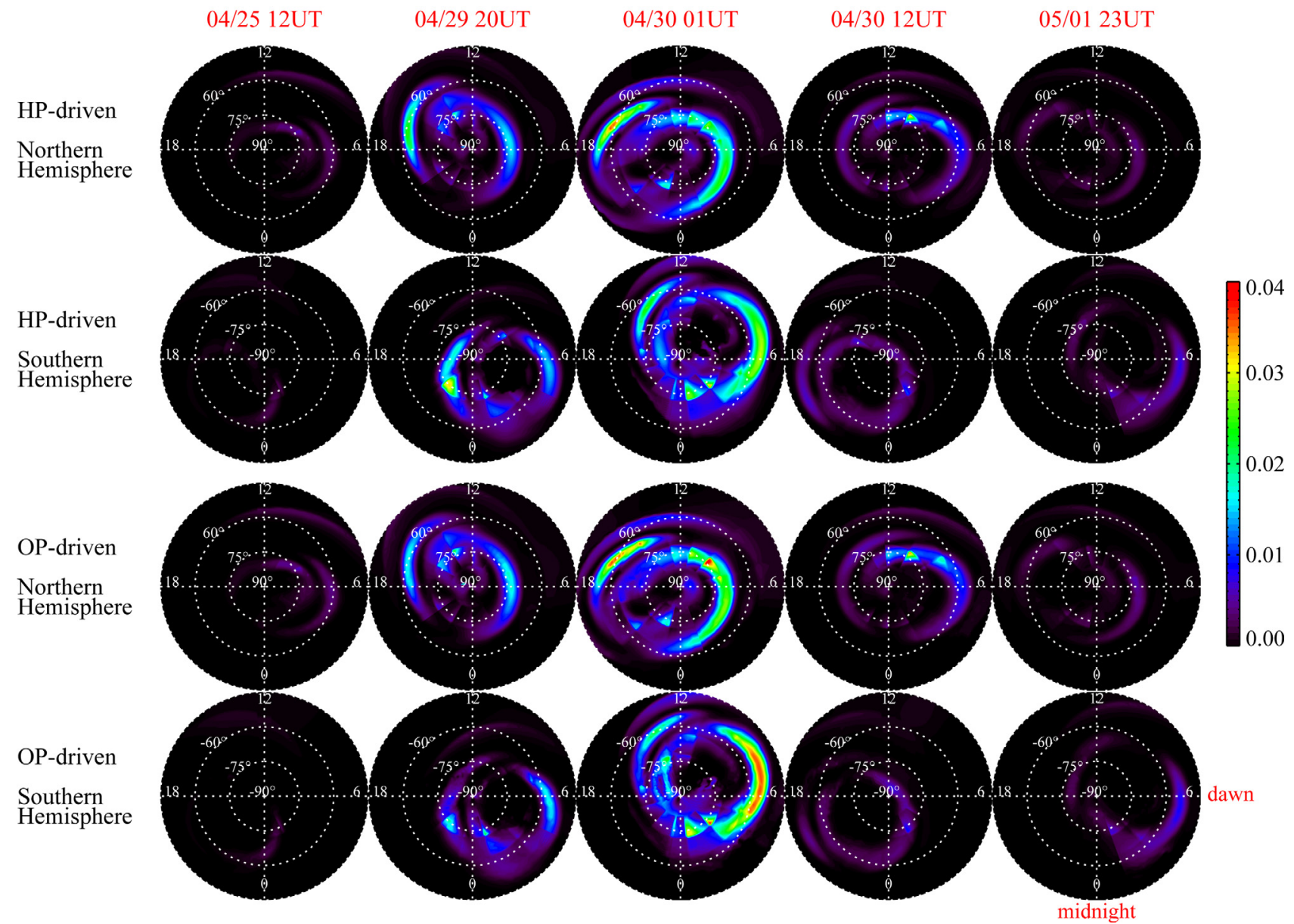

Fig. 9. Height-integrated Joule heating density from the GITM model plotted for the northern hemisphere with the HP driving, for the southern hemisphere with the HP driving, for the northern hemisphere with the OP driving, and for the southern hemisphere with the OP driving (rows from top to bottom). Columns correspond to the times of snapshots shown in Figure 8.

Prime driving shows more detail in the auroral heating pattern during the main phase (bottom panels). Intensifications in auroral heating during the main phase occur at different locations for different models of particle precipitation (on April 30). Note that the OP modeling shows a smaller auroral heating during the secondary peak in the coupling functions at 12:00 UT on April 30 (Figs. 2c-2e) whereas the HP-driven run shows a larger value. Another peak in the coupling functions at 23 UT on 121 DOY (Figs. 2c-2d) does not show itself in the auroral heating and is coincident with minima in HPe and $\mathrm{AE}$ (Figs. $2 \mathrm{j}$ and $2 \mathrm{k}$ ).

\section{Discussion}

In this paper we analyze empirical and GITM modeled parameters which describe coupling, energy transport, and partitioning in the IT system throughout two CIR-HSS events. Solar wind coupling with the IT system increases dramatically at a geomagnetic storm onset when all coupling functions peak. During the HILDCAA interval, the epsilon parameter drops to a pre-event low value, whereas the Universal function and Kan-Lee electric field show efficient coupling with the magnetosphere and even a secondary peak corresponding to the peak solar wind velocity for the 2011 event (Figs. 1 and 2). We should note that only the epsilon parameter is scaled to have the physical meaning of energy. Empirical estimates of Joule heating, observations-derived NO cooling flux, and HPe also show a continuous driving in a storm recovery phase and HSS proper. The fluctuating nature of current dissipation (seen in Joule heating) and HPe could be due to sporadic particle precipitation during HILDCAA (Hajra et al. 2014).

We performed GITM runs driven by the empirical Weiner05 model and AMIE, and different empirical models (NOAA HP and Ovation Prime) for the auroral particle precipitation. Forecasted quantities with a lead time of at least several hours, up to a few days if a heliospheric forecast is available, can be utilized in all inputs, except for NOAA HP. However, HPt or HPe can be estimated from a forecastable $K p$ using $\mathrm{HPt}(\mathrm{GW})=\max (0 .,-2.78+9.33 \mathrm{Kp})$ (Maeda et al. 1989; Emery et al. 2012), $\mathrm{HPe}(\mathrm{GW})=2.5103+9.9207 \mathrm{Kp}-$ $2.1825 K p^{2}+0.3033 K p^{3}$ (Emery et al. 2008), or the estimates of HPt by Zhang \& Paxton (2008) as a function of $K p$ in their Figure 8. Emery et al. (2012) also parameterized the log index HPI as $B_{z}(\mathrm{nT})=3.98-0.78 \times$ HPI using 20 days of data from NOAA-6, 7, 8 and DMSP-F7 satellites between 1981 and 1984, while Emery et al. (2008) found HPe as a function of IMF $B_{z}$, solar wind speed, $10.7 \mathrm{~cm}$ solar flux, and season. Solar EUV and the solar wind environment in principle can be forecasted (Henney et al. 2012; Manchester et al. 2014). The Ovation Prime model is parameterized by the solar wind 


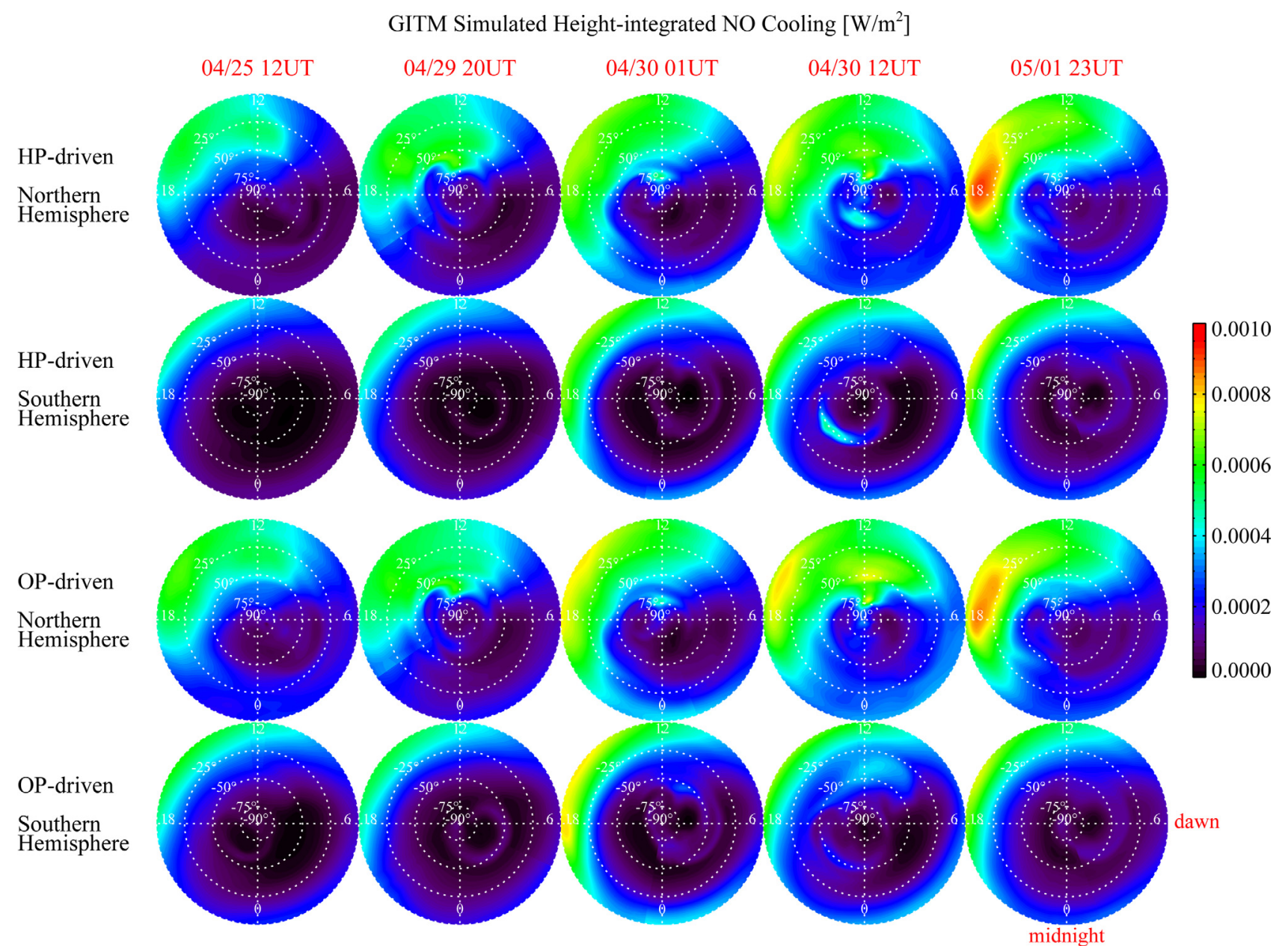

Fig. 10. Height-integrated NO cooling emission power density from the GITM plotted for the northern hemisphere with the HP driving, for the southern hemisphere with the HP driving, for the northern hemisphere with the OP driving, and for the southern hemisphere with the OP driving (rows from top to bottom). Columns correspond to the times of snapshots shown in Figure 8.

and IMF parameters over $4 \mathrm{~h}$ prior. Ovation Prime has been already used to provide forecasts of up to $2 \mathrm{~h}$ lead time (http://www.swpc.noaa.gov/products/30-minute-aurora-forecast). On one hand, auroral heating does not have a major contribution to the IT energy budget in a HSS storm main phase. On the other hand, soft particle precipitation and ionization patterns are important for electron and neutral density estimates especially in high-latitude regions.

The energy budget for the events (Figs. 3 and 8) follows the storm dynamics shown in activity indices and coupling functions. Energy inputs in the main phases of the storms are highly variable at the scale of an hour. Driving by Weimer05 and AMIE results in main phase energy peaks which differ by about a factor of 2 (Figs. $3 \mathrm{~h}$ and $8 \mathrm{~h}$ ). This can be explained by the fact that Joule heating is the dominant energy deposition channel during storm times and is driven by an electrodynamics model. Huang et al. (2012a) have compared modeled Joule heating estimates from AMIE and Weimer05 during HSS intervals for the year 2005. They showed that the Northern hemisphere integrated Joule heating calculated from AMIE is systematically larger than Weimer05 estimates. Sensitivity of Joule heating to solar wind driving is significantly higher through the AMIE procedure than through Weimer05. However, we currently cannot use the AMIE-based technique in forecasting, whereas we can run Weimer05 given forecasted solar wind parameters. Indeed, Joule heating constitutes $\sim 60 \%$ of the total energy budget for the OP-Weimer driven runs during the storm main phases (Figs. $3 \mathrm{~h}$ and $8 \mathrm{~h}$ ), for which values can reach up to $\sim 95 \%$ (but for a CME storm) in another modeling study using AMIE driving (Lu et al. 1995). Our AMIE-driven results are $\sim 92 \%$ and $51 \%$ for peak values at selected snapshots during the main phases for the two events. Deng \& Ridley (2007) addressed the issue of underestimating Joule heating in global coupled IT modeling with the example of GITM. They emphasized importance of feedback from ionospheric electron density and thermospheric neutral winds to Joule heating which both cause additional variability in Joule heating. This is better captured in the current version of GITM.

Different particle precipitation models (HP and OP) do not cause large differences in the total energy budget estimates. Ovation Prime causes stronger NO cooling during the main phase of the storm, and NOAA HP during the recovery phase. According to the modeling results $\mathrm{CO}_{2}$ cooling is the major energy sink for the height-integrated IT energy (Figs. 3 and 8, right-hand panels) and shows little dependence on geomagnetic activity. Total $\mathrm{CO}_{2}$ cooling from the model exceeds modeled NO cooling by about the factor of 5 . This result agrees with SABER measurements (Figs. 1i and 2i) which show that the ratio between $\mathrm{NO}$ and $\mathrm{CO}_{2}$ cooling powers during the storm main phase is about the same. Note that the global power values from SABER were obtained by integrating 
GITM Simulated Height-integrated Auroral Heating [W/m²]

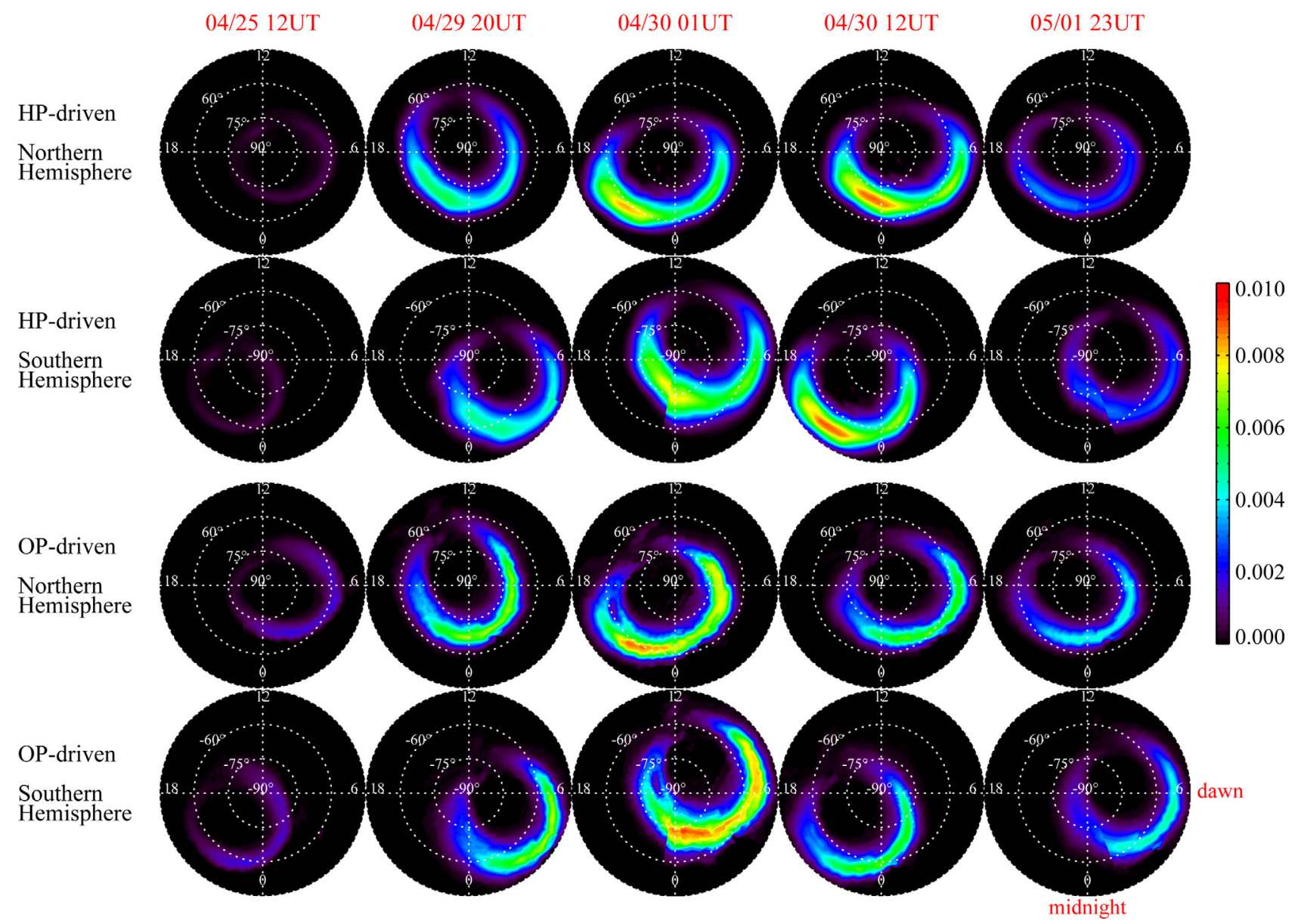

Fig. 11. Height-integrated auroral heating power density from the GITM plotted for the northern hemisphere with the HP driving, for the southern hemisphere with the HP driving, for the northern hemisphere with the OP driving, and for the southern hemisphere with the OP driving (rows from top to bottom). Columns correspond to the times of snapshots shown in Figure 8.

approximately within the altitude range of 100-200 km and GITM values were obtained by integrating up to $600 \mathrm{~km}$.

From the globally averaged height-integrated $\mathrm{NO}$ and $\mathrm{CO}_{2}$ cooling power we estimate the total power by multiplying by the Earth surface area. With the total cooling power during the peak of the 2007 event of $\sim 1.6 \mathrm{~mW} / \mathrm{m}^{2}$ and $\sim 0.2 \mathrm{~mW} / \mathrm{m}^{2}$ for $\mathrm{CO}_{2}$ and NO (Figs. $3 \mathrm{~h}$ and $3 \mathrm{i}$ ), this simple calculation yields $800 \mathrm{GW}$ and $100 \mathrm{GW}$, respectively. This is in reasonably good agreement with SABER-based peak estimates of $\sim 740 \mathrm{GW}$ and $\sim 110 \mathrm{GW}$ (see Fig. 1i).

It is well known that NO cooling is the major energy sink in a certain altitude range, especially around $125 \mathrm{~km}$ altitude. Our modeling results clearly do not support this view (see Figs. 3 and 8, left-hand panels). They show that peak NO cooling fluxes occur in low latitudes during storm recovery phases (Figs. 6 and 10). Observations indicate the highest daily average values of $\sim 0.45 \mathrm{~mW} / \mathrm{m}^{2}$ in the northern winter high latitudes around $70^{\circ}$ for the first event and $\sim 0.76 \mathrm{~mW} / \mathrm{m}^{2}$ in the southern winter high latitudes for the second event. The model peak estimates in the corresponding high-latitude regions are $<0.4 \mathrm{~mW} / \mathrm{m}^{2}$ for the first event and $<0.2 \mathrm{~mW} / \mathrm{m}^{2}$ for the second event. This underestimation of NO cooling could be due to uncertainty in the NO cooling parameterization and NO binary diffusion coefficients in the current version of GITM (Pawlowsky \& Ridley 2009). Detailed comparison of $\mathrm{NO}$ and $\mathrm{CO}_{2}$ cooling model outputs with single scan flux measurements by SABER for these two HSS events is needed to determine possible areas of the model improvement.

Modeled Joule heating at $125 \mathrm{~km}$ shows the same global pattern as the height-integrated quantity. Modeled Joule heating at $300 \mathrm{~km}$ is much smaller (not shown here to save space). This could be an indication that most Joule heating is deposited around $125 \mathrm{~km}$ in agreement with the previous modeling studies (Lu et al. 1995; Weimer 2005; Deng et al. 2011; Huang et al. 2012b). Simple estimates can be done to compare modeled Joule heating in the high-latitude northern hemisphere with empirical values (Figs. 1f and 2f). From Figures 5 and 9 one can see that Joule heating is mostly deposited poleward of the $60^{\circ}$ latitude circle. The corresponding area of the spherical cap is $2 \pi R^{2}\left(1-\sin 60^{\circ}\right)$, where $R$ is the Earth radius. Average modeled Joule heating in the northern hemisphere from Figure 5 is $\sim 0.01 \mathrm{~W} / \mathrm{m}^{2}$ on Jan 29, 2007 at 1400 UT which yields $\sim 340 \mathrm{GW}$ energy input. This value is comparable with the empirical Joule heating of $\sim 300 \mathrm{GW}$ for this time stamp (Fig. 1f). High northern latitude Joule heating is more intense during the main storm phase for the second HSS event (Fig. 9) and the corresponding empirical estimate is also higher (Fig. 2f).

McHarg et al. (2005) analyzed dependence of Joule heating derived from AMIE-based data assimilation on IMF $B_{z}$ orientation. They showed a preference for Joule heating to be located predominantly in the auroral region with increased 
(a)

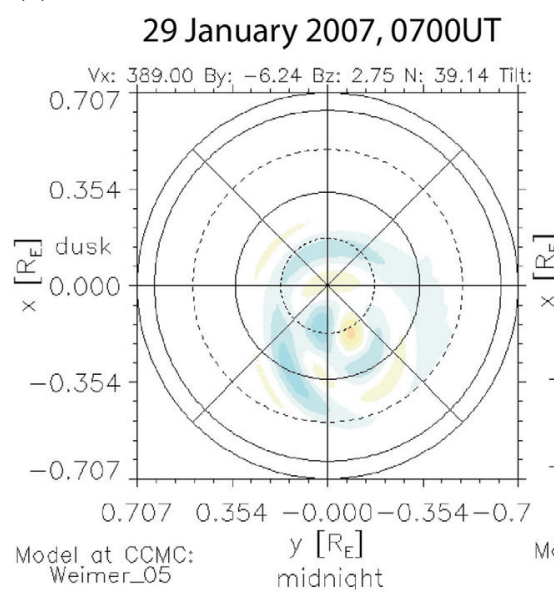

(d)

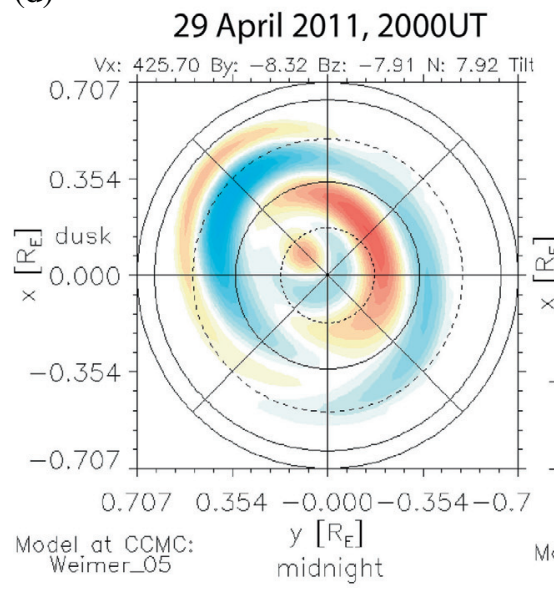

(b)

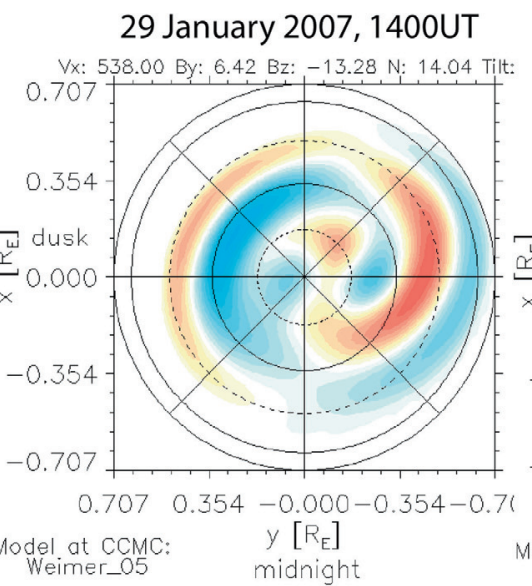

(e) (c)

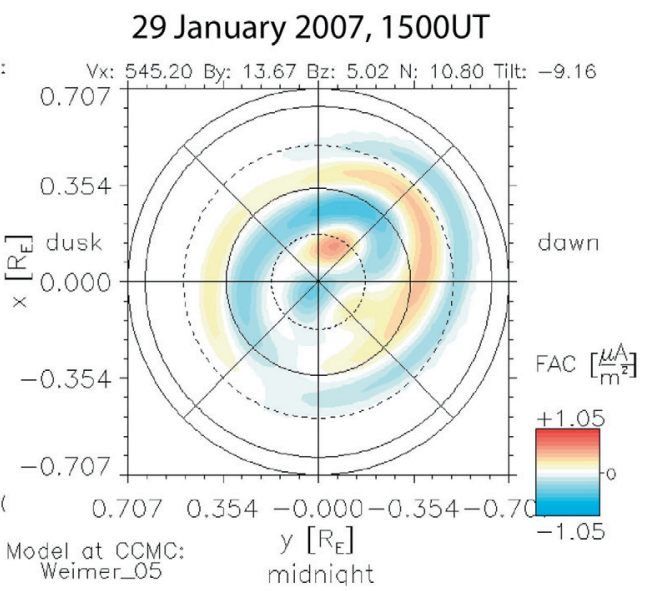

(f)
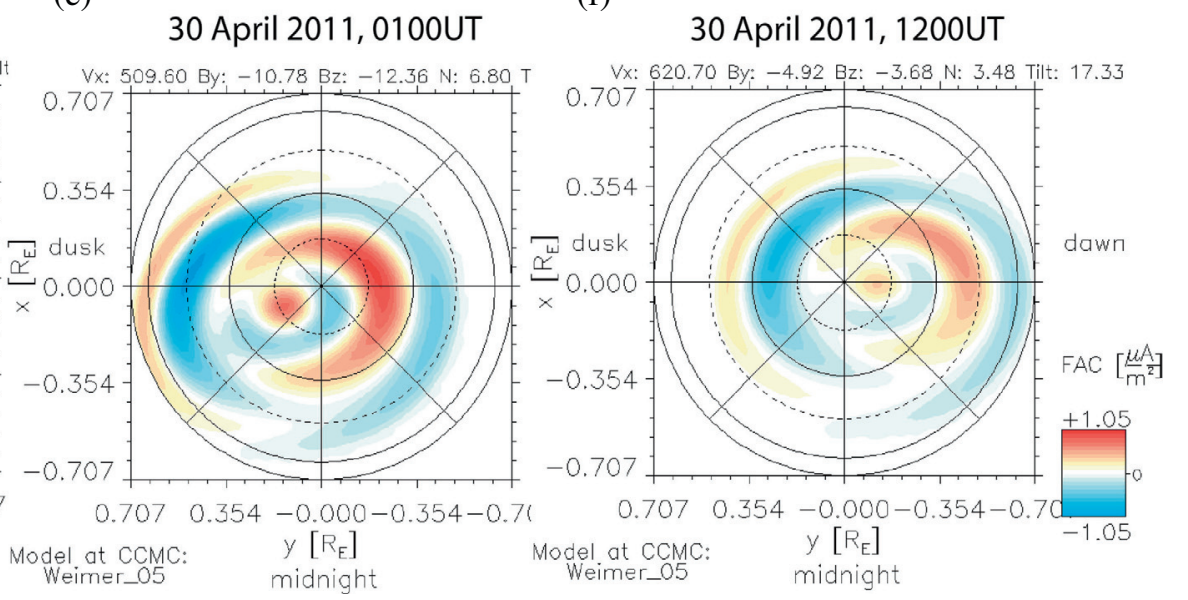

Fig. 12. FACs in the northern hemisphere from the Weimer05 model runs at the CCMC: (a) 29 January 2007, 0700 UT; (b) 29 January 2007, 1400 UT; (c) 29 January 2007, 1500 UT; (d) 29 April 2011, 2000 UT; (e) 30 April 2011, 0100 UT; (f) 30 April 2011, 1200 UT. Positive (red) current is downward and negative (blue) current is upward.

heating in the morning sector compared to the evening sector and strong local time asymmetry during southward IMF conditions. McHarg et al. (2005) also noted an increase in the daytime southern high-latitude region. Our modeling results show a preference toward the morning sector and $60^{\circ}$ to $75^{\circ}$ latitude range in the northern hemisphere, and toward the morning and sometimes the afternoon sector within $-75^{\circ}$ to $-60^{\circ}$ latitude range in the southern hemisphere. Thus, the GITM modeling results are consistent with the previous observations.

How do we relate Weimer05 input and especially fieldaligned current (FAC) with modeled Joule heating latitudinal and local time patterns? From first-principle-physics we would expect that Joule heating is mostly deposited in the auroral ionosphere where Region 1 (R1) and Region 2 (R2) currents close (Kelley 2009, Fig. 8.2). The R1 current system is more poleward than the R2 current system and it flows into the ionosphere in the polar cap. R1 current is typically downward in the dawn sector and upward in the dusk sector in the northern hemisphere. R2 current has the reverse direction in these sectors. Figure 12 (top row) presents several snapshots of the Weimer05 potential for the northern hemisphere during the 2007 HSS event which is used for high-latitude external driving of our model. These maps are made using the real-time run interface at the Community Coordinated Modeling Center (CCMC) (http://ccmc.gsfc.nasa.gov/cgi-bin/run_weimer.cgi) with solar wind parameters from the OMNI database as inputs. Times correspond to the main phase and recovery phase snapshots in Figure 5. Latitudinal circles are drawn every $10^{\circ}$. Positive (red) current is downward (Weimer 2005). For the snapshot on January 29 at 0700 UT the FAC pattern is relatively weak (Fig. 12a) and the pre-noon sector resembles the Joule heating pattern in Figure $5 \mathrm{~b}$ (top row). During the storm main phase the FAC pattern intensifies (Fig. 12b) and shows a pronounced dawn-side downward current around $60^{\circ}$ latitude. Joule heating is intensified approximately equatorward from that arc (Fig. 5c, top row). Note weaker Joule heating arcs which are located approximately close to intense FAC regions in the dayside auroral zone. An hour later, FAC became weaker and more dayside (Fig. 12c). These features are similar to the corresponding Joule heating pattern (Fig. 5d, top row).

Figure 12 (bottom row) presents several snapshots of the Weimer05 FAC for the northern hemisphere for the 2011 event corresponding to time snapshots of Figures 9b-9d (top row). Again, there are similarities between the FAC arcs and the Joule heating intensification pattern. Generally, Joule heating is intensified toward the equatorward edge of downward (red) FAC current on either the dawn-side or day-side (compare Figs. 9c and 12e). This is consistent with the equatorward edge of the R1 current and a region in the ionosphere where R1 and $\mathrm{R} 2$ close. 
The FAC system has typical temporal variability scales of $\sim 40-60 \mathrm{~s}$ on the dayside and $\sim 90-140 \mathrm{~s}$ on the nightside (Gjerloev et al. 2011). Thus, IT modeling based on models of FAC may not be able to capture the short-time and smallscale variability of the Joule heating patterns. Note that the solar wind clock angle, between IMF $B_{y}$ and IMF $B_{z}$, controls local time locations of intense FACs (see Fig. 4 in Weimer 2005) and, correspondingly, locations of intense Joule heating arcs. One of the limitations of the Weimer05 model is that it does not include cusp heating or heating outside the auroral area. We would like to note that modeled Joule heating and NO cooling patterns show strong inter-hemispheric asymmetry for the 2007 event and a lesser inter-hemispheric symmetry for the 2011 event. Interestingly, the first event was characterized by large positive IMF $B_{y}$ and the second event was characterized by large negative IMF $B_{y}$ during the selected snapshots.

Particle precipitation in our modeling is driven by either NOAA HP or OP. Intensities of modeled auroral heating for these two cases are rather different but locations are close (Figs. 7 and 11). Statistical analysis based on 28 days of DMSP satellite energy flux measurements shows that OP can accurately describe the location of the auroral oval's equatorward boundary (Lane et al. 2015). Auroral electron precipitation is expected to occur near upward R1, especially during intervals of IMF $B_{z}<0$ (Ohtani et al. 2010; Korth et al. 2014). Careful comparison of auroral heating maps (Figs. 7 and 11, top plots) with FACs (Fig. 12) indicates that auroral heating arcs are located approximately in the vicinity of the upward (negative, shown in blue) currents in the auroral region. Note enhanced electron precipitation in the morning sector (Fig. 7) occurring at the main phase onset of the 2007 storm on January 29, $1500 \mathrm{UT}$ in the NH for OP and especially HP. This auroral heating intensification is probably due to electron interaction with the chorus waves and acceleration (Tsurutani et al. 2006). Simple estimation shows that auroral heating flux from the region between $60^{\circ}$ and $75^{\circ}$ during the main phase of the 2007 storm is $\sim 2 \pi R^{2}\left(\sin 75^{\circ}-\sin 60^{\circ}\right) 5 \mathrm{~mW} /$ $\mathrm{m}^{2} \approx 170 \mathrm{GW}$, which agrees with empirical HPe peaks (Fig. 1j).

HPe shows temporal periodicities and variations which are controlled (at $\sim 4 \%$ ) by the solar wind velocity (Emery et al. 2009). Combined use of NOAA POES and DMSP data improved resolution of the spatial inhomogeneity of the electron precipitation pattern. There are well-known uncertainties in determining HP, see for instance the discussion in Emery et al. $(2009,2011)$. There is a need of extended climatology reference maps for total energy high-latitude flux patterns beyond several years (Fuller-Rowell \& Evans 1987) and preferably covering different phases of a solar cycle.

Interestingly, the NOAA HP driven run produces NO cooling emission almost twice as strong as the OP-driven run for the 2007 storm, especially in the main phase. On the other hand, there is larger NO cooling for the OP-driven run during the weaker but prolonged 2011 storm than for the OP-driven run for the 2007 storm, especially during the recovery phase.

In general, peak model values of Joule heating and auroral heating agree with observational proxies and first-principlesphysics, even though the empirical Weimer05 model cannot capture small-scale variability of the Joule heating pattern. It is difficult to estimate the Joule heating rate since both electrodynamics (through electric field and conductance) and neutral dynamics (through winds) contribute to it (Thayer et al. 1995). Using Poynting flux might be a better way to estimate
Joule heating based on formula (1). It is an important characteristic of magnetosphere-IT coupling during HSS intervals. Knipp et al. (2011) have shown that Poynting flux derived from DMSP measurements during intervals of large IMF $B_{y}$ and HSSs exceeded super-storm values (Huang \& Burke 2004). Statistical analysis of DMSP measurements binned by geomagnetic storm intensity shows that Poynting flux values during moderate $(-200 \mathrm{nT}<\mathrm{SYM}-\mathrm{H}<-100 \mathrm{nT})$ and weak storms (SYM-H $>-100 \mathrm{nT}$ ) are comparable (Huang, Y., personal communication, 2015). However, one needs to take caution under which conditions Poynting flux perturbations above the ionosphere, as obtained from the cross product of the electric and magnetic perturbation fields observed on a spacecraft, may be used to estimate electromagnetic energy dissipation in the ionosphere (Richmond 2010). Another caveat in estimating energy input into the IT system is taking into account transfer through very high-latitude regions (Huang et al. 2014) which is not accounted for in the Weimer05 model, for instance. This is the subject of further modeling development.

The total (height-integrated) heating and cooling do not balance in the model. Storm-time heating in the modeling is dominated by Joule heating, while the model cooling is dominated by $\mathrm{CO}_{2}$ and not dependent on geomagnetic activity. We suggest that the lower boundary driving at $100 \mathrm{~km}$ could be the reason for the overestimated $\mathrm{CO}_{2}$ cooling which originates around $100 \mathrm{~km}$. The MSIS model was used to provide an estimate for the atmospheric temperature at the GITM lower boundary of $\sim 100 \mathrm{~km}$ (Ridley et al. 2006; Vichare et al. 2012). In the course of modeling GITM estimates the temperatures in adjacent altitude computational layers. However, if the MSIS does not represent the lower boundary conditions well, this could result in increased cooling near $100 \mathrm{~km}$ altitude in the simulations, even during quiet-time conditions (see Figs. $3 \mathrm{f}$ and 8f).

The dynamics of these two HSS events are very similar. More energy (estimate of height-integrated power for the OP run is $\sim 4 \mathrm{~mW} / \mathrm{m}^{2}$ ) seems to enter the IT system during the 2011 event than during the 2007 event (height-integrated for the OP run $\sim 3 \mathrm{~mW} / \mathrm{m}^{2}$ ). However, the ratio of total heating power to a pre-event background heating power is about the same for the two events $\sim 3.6$ and 3.9 for the 2011 and 2007 events, correspondingly.

\section{Conclusions}

We analyze solar wind-ionosphere coupling and IT dynamics during CIR-HSS sub-intervals within 22-31 January, 2007 and 25 April-2 May, 2011. We discuss a correspondence between the solar-wind - magnetosphere coupling functions, estimated IT energy inputs, and partitioning inside the IT system (Joule heating and auroral heating). The coupling functions provide physics-based adequate descriptions of energy input into the IT system and prolonged coupling during the HILDCAA interval. One possible shortcoming of the way the coupling functions are defined is that they are derived mostly from measurements at the L1 point and thus we ignore the three-dimensional nature of the solar wind - magnetosphere interactions. Measurements at L1 and L5 points together could have important implications for better understanding the coupling and for forecasting (Vourlidas 2015).

We performed GITM runs driven by the Weimer05 highlatitude electrodynamics model or in two cases by AMIE, 
and two models of particle precipitation. The energy budget and energy partitioning in our modeling are in agreement with empirical estimates and the previous studies Turner et al. (2009) and Hajra et al. (2014) with the exception of cooling mechanisms. The total energy budget is mostly driven by Joule heating and shows little dependence on the particle precipitation model. The ratio of total event heating power in a storm main phase to pre-event background heating power is about 3.6 to 4 for these events.

These are the main results of the GITM modeling:

- Joule heating values generally agree with empirical estimates. The local time pattern agrees with the statistical study of AMIE Joule heating patterns by McHarg et al. (2005). Joule heating is highly variable during the storm main phase at scales $\leq 1 \mathrm{~h}$ and is dependent on Weimer05 or AMIE driving. The inter-hemispheric asymmetry of the Joule heating pattern in the dawn or afternoon regions is possibly controlled by the interplanetary magnetic field clock angle. We would like to suggest that Poynting flux estimates from in situ measurements could be the most accurate way of estimating Joule heating. However, the impact of limited sampling from an in situ measurement needs to be evaluated.

- Modeled Joule heating intensification patterns from Weimer05 driving in the Northern hemisphere agree with Weimer05 FACs and are controlled by IMF $B_{y}$ and $B_{z}$.

- NO model cooling is largest in the summer dayside in middle to low latitudes in the afternoon (Figs. 6 and 10) while SABER observations show the largest cooling values in the winter high latitudes (Figs. 1 and 2, panels $g$ and $h$ ) such that the height-integrated NO cooling in the model is underestimated during the storm (Meng et al., paper in preparation).

- Globally averaged NO and CO2 cooling powers show good agreement with SABER measurements.

- The auroral heating shows similar local time patterns but different intensity peaks in time for OP and NOAA HP controlled runs. Auroral heating generally agrees with the HP estimate during storm events. Inter-hemispheric asymmetry of auroral heating changes throughout storm phases and from event to event.

We performed GITM runs with Weimer05 in a forecasting mode, i.e., without tuning model parameters and with inputs (solar UV flux, solar wind parameters, and activity indices) which are in principle forecastable at the current state of global model development. This was done to assess feasibility of IT forecasting. We believe that the modeling captures the main features of the energy input and, thus, the physics of magnetosphere-IT coupling. More effort is needed to adequately model thermospheric cooling. Coupling from below can be very important for estimating the total energy balance in the IT system. Vichare et al. (2012) have shown that the choice of a model for lower boundary tidal forcing impacts GITM modeling results on ionospheric plasma drifts and electron density.

In our opinion there are several challenges in developing forecasting capabilities by using global models:

1. Physical inputs which can substantially improve forecasting capability need to be identified and means of their forecasting should be studied. Uncertainty in these parameters will affect the robustness of the prediction.
2. There are limitations on scales of temporal and spatial variability of the IT system that can be forecast. They need to be defined.

3. The role of pre-conditioning for IT system responses to geomagnetic activity needs to be further analyzed. This issue is related to intrinsic predictability of global IT.

4. Optimal validation databases need to be identified. We need to determine which observations would provide crucial contributions to understanding the physics behind IT forecasting. For instance, Poynting flux measurements can be very important for our understanding of forecasting capabilities in the energy domain.

5. Forecastable quantities of IT need to be evaluated based on their importance for applications and on their potential predictability.

6. Analyses of unsuccessful or "far-off" forecasts are equally important. They will provide insight into why models do not capture each event and what needs to be improved.

Acknowledgements. Portions of this work were done at the Jet Propulsion Laboratory, California Institute of Technology, under a contract with NASA. Sponsorship of the Heliophysics Division of the NASA Science Mission Directorate is gratefully acknowledged. MGM would like to acknowledge support from the NASA TIMED project office. RH would like to thank Brazilian FAPESP agency for financial support. The computing resources were provided by the NASA High-End Computing Program through the NASA Advanced Supercomputing Division at Ames Research Center. SABER data are available at http://saber.gats-inc.com/. Solar wind parameters and activity indices are taken from the OMNI database (http://omniweb.gsfc.nasa.gov/form/omni_min.html). This study used indices from the CEDAR Database at the National Center for Atmospheric Research which is supported by the National Science Foundation. The instant run facility of CCMC was used for the Weimer05 model runs. Solar radiance was taken from the Space Physics Interactive Data Resource (http://spidr.ngdc.noaa.gov/ spidr/home.do). Flare Irradiance Spectral Model (FISM) provided empirical solar irradiance spectra (http://lasp.colorado.edu/lisird/ fism/). The ground magnetometer data was downloaded from the SuperMAG website: http://supermag.jhuapl.edu/). X. Meng would like to thank A. Ridley (University of Michigan) and Y. Deng (University of Texas at Arlington, TX) for consulting on the GITM. OV would like to thank D. Knipp (University of Colorado, Boulder) and Ja Soon Shim (NASA GSFC) for Poynting flux data samples. The editor thanks two anonymous referees for their assistance in evaluating this paper.

\section{References}

Akasofu, S.-I. Interplanetary energy flux associated with magnetospheric substorms. Planet. Space Sci., 27, 425-431, 1979. DOI: 10.1016/0032-0633(79)90119-3.

Burke, W.J., D.R. Weimer, and N.C. Maynard. Geoeffective interplanetary scale sizes derived from regression analysis of polar cap potentials. J. Geophys. Res., 104, 9989-9994, 1999, DOI: $10.1029 / 1999 J A 900031$

Burke, W.J., L.C. Gentile, and M.P. Hagan. Thermospheric heating by high-speed streams in the solar wind. J. Geophys. Res., 115, A06318, 2010, DOI: 10.1029/2009JA014585.

Burns, A.G., S.C. Solomon, L. Qian, W. Wang, B.A. Emery, M. Wiltberger, and D.R. Weimer. The effects of corotating interaction region/High speed stream storms on the thermosphere and ionosphere during the last solar minimum. J. Atmos. Sol. Terr. Phys., 83, 79-87, 2012, DOI: 10.1016/j.jastp.2012.02.006.

Burton, R.K., R.L. McPherron, and C.T. Russell. An empirical relationship between interplanetary conditions and Dst. $J$. 
Geophys. Res., 80 (31), 4204-4214, 1975, DOI: $10.1029 / \mathrm{JA} 080 \mathrm{i} 031 \mathrm{p} 04204$.

Chamberlin, P.C., T.N. Woods, and F.G. Eparvier. Flare Irradiance Spectral Model (FISM): daily component algorithms and results. Space Weather, 5, S07005, 2007.

Cole, K.D. Joule heating of the upper atmosphere. Australian J. Phys., 15, 223-235, 1961.

Deng, Y., and A.J. Ridley. Possible reasons for underestimating Joule heating in global models: E field variability, spatial resolution, and vertical velocity. J. Geophys. Res., 112, A09308, 2007, DOI: 10.1029/2006JA012006.

Deng, Y., Y. Huang, J. Lei, A.J. Ridley, R. Lopez, and J. Thayer. Energy input into the upper atmosphere associated with highspeed solar wind streams in 2005. J. Geophys. Res., 116, A05303, 2011, DOI: 10.1029/2010JA016201.

Drob, D.P., J.T. Emmert, G. Crowley, J.M. Picone, G.G. Shepherd, et al. An empirical model of the Earth's horizontal wind fields: HWM07. J. Geophys. Res., 113, A12304, 2008, DOI: 10.1029/2008JA013668.

Echer, E., B.T. Tsurutani, and W.D. Gonzalez. Interplanetary origins of moderate $(-100 \mathrm{nT}<\mathrm{Dst}<-50 \mathrm{nT})$ geomagnetic storms during solar cycle 23 (1996-2008). J. Geophys. Res., 118, 385-392, 2013, DOI: 10.1029/2012JA018086.

Emery, B.A., D.S. Evans, M.S. Greer, E. Holeman, K. KadinskyCade, F.J. Rich, and W. Xu. The low energy auroral electron and ion hemispheric power after NOAA and DMSP intersatellite adjustments. NCAR technical note, NCAR/TN-470+STR, HAO/ NCAR, 2006

Emery, B.A., V. Coumans, D.S. Evans, G.A. Germany, M.S. Greer, E. Holeman, K. Kadinsky-Cade, F.J. Rich, and W. Xu. Seas onal, $\mathrm{Kp}$, solar wind, and solar flux variations in long-term single-pass satellite estimates of electron and ion auroral hemispheric power. J. Geophys. Res., 113, 2008, A06311, DOI: 10.1029/2007JA012866.

Emery, B.A., I.G. Richardson, D.S. Evans, and F.J. Rich. Solar wind structure sources and periodicities of auroral electron power over three solar cycles. J. Atmos. Sol. Terr. Phys., 71, 2009, 1157-1175, DOI: 10.1016/j.jastp.2008.08.005.

Emery, B.A., I.G. Richardson, D.S. Evans, F.J. Rich, and G.R. Wilson. Solar rotational periodicities and semiannual variation in the solar wind, radiation belt, and aurora. Solar Physics, 274, 399-425, 2011, DOI: 10.1007/s11207-011-9758-x.

Emery, B.A., R.G. Roble, E.C. Ridley, A.D. Richmond, D.J. Knipp, G. Crowley, D.S. Evans, F.J. Rich, and S. Maeda. Parameterization of the ion convection and the auroral oval in the NCAR thermospheric general circulation models, NCAR technical note, NCAR/TN-491+STR, HAO/NCAR, 2012.

Foster, J.C., J.M. Holt, R.G. Musgrove, and D.S. Evans. Ionospheric convection associated with discrete levels of particle precipitation. Geophys. Res. Lett., 13, 656, 1986 , DOI: $10.1029 / \mathrm{GL} 013 \mathrm{i} 007 \mathrm{p} 00656$

Fuller-Rowell, T.J., and D.S. Evans. Height-integrated Pedersen and Hall conductivity patterns inferred from the TIROS/NOAA satellite data. J. Geophys. Res., 92, 7606-7618, 1987, DOI: 10.1029/JA092iA07p07606.

Gjerloev, J.W., S. Ohtani, T. Iijima, B. Anderson, J. Slavin, and G. Le. Characteristics of the terrestrial field-aligned current system. Ann. Geophys., 29, 1713-1729, 2011,

DOI: 10.5194/angeo-29-1713-2011.

Gonzalez, W.D., and B.T. Tsurutani. Criteria of interplanetary parameters causing intense magnetic storms. Planet. Space Sci., 35 (9), 1101-1109, 1987,

DOI: 10.1016/0032-0633(87)90015-8.

Gonzalez, W.D., J.A. Joselyn, Y. Kamide, H.W. Kroehl, G. Rostoker, B.T. Tsurutani, and V.M. Vasyliunas. What is a geomagnetic storm? J. Geophys. Res., 99, 5771, 1994,

DOI: 10.1029/93JA02867.

Gonzalez, W.D., F.L. Guarnieri, A.L. Clua-Gonzalez, E. Echer, and M.V. Alves. Magnetospheric energetics during HILDCAAs. In: B. Tsurutani, R. McPherron, G. Lu, J.H.A. Sobral, and
N. Gopalswamy, Editors. Recurrent Magnetic Storms: Corotating Solar Wind Streams, American Geophysical Union, Washington, DC, 2006, DOI: 10.1029/167GM15.

Guarnieri, F.L. The nature of auroras during high-intensity longduration continuous AE activity (HILDCAA) events, 1998 to 2001. In: B. Tsurutani, R. McPherron, G. Lu, J.H.A. Sobral, and N. Gopalswamy, Editors. Recurrent Magnetic Storms: Corotating Solar Wind Streams, American Geophysical Union, Washington, DC, 2006, DOI: 10.1029/167GM19.

Hagan, M., K. Häusler, G. Lu, J.M. Forbes, and X. Zhan. Upper thermospheric responses to forcing from above and below during April 1-10, 2010: results from an ensemble of numerical simulations. J. Geophys. Res., 120, 3160-3174, 2015, DOI: $10.1002 / 2014 J A 020706$.

Hajra, R., E. Echer, B.T. Tsurutani, and W.D. Gonzalez. Solar cycle dependence of high-intensity long-duration continuous AE activity (HILDCAA) events, relativistic electron predictors? J. Geophys. Res., 118, 5626-5638, 2013, DOI: 10.1002/jgra.50530.

Hajra, R., E. Echer, B.T. Tsurutani, and W.D. Gonzalez. Solar windmagnetosphere energy coupling efficiency and partitioning: HILDCAAs and preceding CIR-storms during solar cycle 23. J. Geophys. Res., 119, 2675-2690, 2014, DOI: $10.1002 / 2013 J A 019646$.

Hardy, D.A., E.G. Holeman, W.J. Burke, L.C. Gentile, and K.H. Bounar. Probability distributions of electron precipitation at high magnetic latitudes. J. Geophys. Res., 113, A06305, 2008, DOI: $10.1029 / 2007$ JA012746.

Hedin, A. Extension of the MSIS thermosphere model into the middle and lower atmosphere. J. Geophys. Res., 96, 1159-1172, 1991, DOI: 10.1029/90JA02125.

Heelis, R.A. Aspects of Coupling Processes in the Ionosphere and Thermosphere. In: J. Huba, R. Schunk, and G. Khazanov, Editors. Modeling the Ionosphere-Thermosphere System, John Wiley \& Sons, Ltd, Chichester, UK, 2013,

DOI: $10.1002 / 9781118704417 . c h 14$.

Henney, C.J., W.A. Toussaint, S.M. White, and C.N. Arge. Forecasting F10.7 with solar magnetic flux transport modeling. Space Weather, 10, S02011, 2012,

DOI: $10.1029 / 2011$ SW000748.

Huang, Y., Y. Deng, J. Lei, A. Ridley, R. Lopez, R.C. Allen, and B. MacButler. Comparison of Joule heating associated with highspeed solar wind between different models and observations. J. Atmos. Sol. Terr. Phys., 75-76, 5-14, 2012a, DOI: 10.1016/j.jastp.2011.05.013.

Huang, Y., A.D. Richmond, Y. Deng, and R. Roble. Height distribution of Joule heating and its influence on the thermosphere. J. Geophys. Res., 117, A08334, 2012b, DOI: $10.1029 / 2012 J A 017885$.

Huang, C.Y., and W.J. Burke. Transient sheets of field-aligned current observed by DMSP during the main phase of a magnetic superstorm. J. Geophys. Res., 109, A06303, 2004, DOI: $10.1029 / 2003 J A 010067$.

Huang, C.Y., Y.-J. Su, E.K. Sutton, D. R.Weimer, and R.L. Davidson. Energy coupling during the August 2011 magnetic storm. J. Geophys. Res., 119, 1219-1232, 2014, DOI: $10.1002 / 2013 J A 019297$.

Kan, J.R., and L.C. Lee. Energy coupling and the solar wind dynamo. Geophys. Res. Lett., 6, 577-580, 1979, DOI: $10.1029 / \mathrm{GL} 006 \mathrm{i} 007 \mathrm{p} 00577$.

Katus, R.M., and M.W. Liemohn. Similarities and differences in low- to middle-latitude geomagnetic indices. J. Geophys. Res., 118, 5149-5156, 2013, DOI: 10.1002/jgra.50501.

Kelley, M.C. The Earth's ionosphere: plasma physics and electrodynamics. 2nd edition, Elsevier, Burlington, MA 01803, USA, 556,2009

Knipp, D.J., W.K. Tobiska, and B.A. Emery. Direct and indirect thermospheric heating sources for the solar cycle 21-23. Solar Physics, 224, 495-505, 2004, DOI: $10.1007 / \mathrm{s} 11207-005-6393-4$ 
Knipp, D.J., T. Welliver, M.G. McHarg, F.K. Chun, W.K. Tobiska, and D. Evans. Climatology of extreme upper atmospheric heating events. Adv. Space Res., 36, 2506-2510, 2005.

Knipp, D., S. Eriksson, L. Kilcommons, G. Crowley, J. Lei, M. Hairston, and K. Drake. Extreme Poynting flux in the dayside thermosphere: examples and statistics. Geophys. Res. Lett., 38, L16102, 2011, DOI: 10.1029/2011GL048302.

Korth, H., Y. Zhang, B.J. Anderson, T. Sotirelis, and C.L. Waters. Statistical relationship between largescale upward field-aligned currents and electron precipitation. J. Geophys. Res., 119, 6715-6731, 2014, DOI: 10.1002/2014JA019961.

Koskinen, H.E.J., and E. Tanskanen. Magnetospheric energy budget and the epsilon parameter. J. Geophys. Res., 107 (A11), 1415, 2002, DOI: 10.1029/2002JA009283.

Kozyra, J.U., G. Crowley, B.A. Emery, X. Fang, G. Maris, et al. Response of the upper/middle atmosphere to coronal holes and powerful high-speed solar wind streams in 2003. In: B. Tsurutani, R. McPherron, G. Lu J.H.A. Sobral, and N. Gopalswamy, Editors. Recurrent Magnetic Storms: Corotating Solar Wind Streams, American Geophysical Union, Washington, DC, 2006, DOI: $10.1029 / 167 \mathrm{GM} 24$

Lane, C., A. Acebal, and Y. Zheng. Assessing predictive ability of three auroral precipitation models using DMSP energy flux. Space Weather, 13, 61-71, 2015, DOI: 10.1002/2014SW001085.

Liu, H.-L., W. Wang, A.D. Richmond, and R.G. Roble. Ionospheric variability due to planetary waves and tides for solar minimum conditions. J. Geophys. Res., 115, A00G01, 2010, DOI: 10.1029/2009JA015188.

Lu, G. High-speed streams, coronal mass ejections, and interplanetary shocks: a comparative study of geoefectiveness. In: B. Tsurutani, R. McPherron, G. Lu, J.H.A. Sobral, and N. Gopalswamy, Editors. Recurrent Magnetic Storms: Corotating Solar Wind Streams, American Geophysical Union, Washington, DC, 2006, DOI: 10.1029/167GM10.

Lu, G., A.D. Richmond, B.A. Emery, and R.G. Roble. Magnetosphere-ionosphere-thermosphere coupling: effect of neutral winds on energy transfer and field-aligned current. J. Geophys. Res., 100 (A10), 19643-19659, 1995, DOI: 10.1029/95JA00766.

Lu, G., M.G. Mlynczak, L.A. Hunt, T.N. Woods, and R.G. Roble. On the relationship of Joule heating and nitric oxide radiative cooling in the thermosphere. J. Geophys. Res., 115, A05306, 2010, DOI: 10.1029/2009JA014662.

Maeda, S., T.J. Fuller-Rowell, and D.S. Evans. Zonally averaged dynamical and compositional response of the thermosphere to auroral activity during September 18-24, 1984. J. Geophys. Res., 94, 16869-16883, 1989, DOI: 10.1029/JA094iA12p16869.

Manchester IV, W.B., B. van der Holst, and B. Lavraud. Flux rope evolution in interplanetary coronal mass ejections: the 13 May 2005 event. Plasma Phys. Control. Fusion, 56 (6), 064006, 2014.

Mannucci, A.J., O.P. Verkhoglyadova, B.T. Tsurutani, X. Meng, X. $\mathrm{Pi}$, et al. Medium-Range Thermosphere-Ionosphere Storm Forecasts. Space Weather, 13, 125-129, 2015, DOI: $10.1002 / 2014$ SW001125.

McHarg, M., F. Chun, D. Knipp, G. Lu, B. Emery, and A. Ridley. High-latitude Joule heating response to IMF inputs. J. Geophys. Res., 110, A08309, 2005, DOI: 10.1029/2004JA010949.

Mlynczak, M., F.J. Martin-Torres, J.M. Russell, K. Beaumont, S. Jacobson, et al. The natural thermostat of nitric oxide emission at $5.3 \mathrm{~mm}$ in the thermosphere observed during the solar storms of April 2002. Geophys. Res. Lett., 30, 2100, 2003, DOI: $10.1029 / 2003$ GL017693.

Mlynczak, M.G., F.J. Martin-Torres, C.J. Mertens, B.T. Marshall, R.E. Thompson, et al. Solar-terrestrial coupling evidenced by periodic behavior in geomagnetic indexes and the infrared energy budget of the thermosphere. Geophys. Res. Lett., 35, L05808, 2008, DOI: 10.1029/2007GL032620.

Mlynczak, M.G., L.A. Hunt, B.T. Marshall, F.J. Martin-Torres, C.J. Mertens., et al. Observations of infrared radiative cooling in the thermosphere on daily to multiyear timescales from the TIMED/
SABER instrument. J. Geophys. Res., 115, A03309, 2010a, DOI: $10.1029 / 2009 J A 014713$.

Mlynczak, M.G., L.A. Hunt, J.U. Kozyra, and J.M. Russell III. Short-term periodic features observed in the infrared cooling of the thermosphere and in solar and geomagnetic indexes from 2002 to 2009. Proc. R. Soc. A, 466, 3409-3419, 2010b,

DOI: $10.1098 /$ rspa.2010.0077.

Newell, P.T., T. Sotirelis, K. Liou, C.-I. Meng, and F.J. Rich. A nearly universal solar wind-magnetosphere coupling function inferred from 10 magnetospheric state variables. J. Geophys. Res., 112, A01206, 2007, DOI: 10.1029/2006JA012015.

Newell, P.T., T. Sotirelis, and S. Wing. Diffuse, monoenergetic, and broadband aurora: the global precipitation budget. J. Geophys. Res., 114, A09207, 2009, DOI: 10.1029/2009JA014326.

Ohtani, S., S. Wing, P.T. Newell, and T. Higuchi. Locations of nightside precipitation boundaries relative to $\mathrm{R} 2$ and $\mathrm{R} 1$ currents. J. Geophys. Res., 115, A10233, 2010, DOI: $10.1029 / 2010 J A 015444$

Pawlowsky, D.J., and A.J. Ridley. Quantifying the effect of thermospheric parameterization in a global model. J. Atmos. Sol. Terr. Phys., 71, 2017-2026, 2009, DOI: $10.1016 /$ j.jastp.2009.09.007.

Perreault, P., and S.-I. Akasofu. A study of geomagnetic storm. Geophys. J. Int., 54, 547-573, 1978.

Richmond, A.D. On the ionospheric application of Poynting's theorem. J. Geophys. Res., 115, A10311, 2010, DOI: $10.1029 / 2010 J A 015768$

Richmond, A., and Y. Kamide. Mapping electrodynamic features of the high-latitude ionosphere from localized observations: technique. J. Geophys. Res., 93, 5741-5759, 1988, DOI: $10.1029 / \mathrm{JA} 093 \mathrm{iA06p05741.}$

Ridley, A.J., and E.A. Kihn. Polar cap index comparisons with AMIE cross polar cap potential, electric field, and polar cap area. Geophys. Res. Lett., 31, L07801, 2004, DOI: 10.1029/2003GL019113.

Ridley, A.J., Y. Deng, and G. Toth. The global ionospherethermosphere model. J. Atmos. Sol. Terr. Phys., 68, 839-864, 2006, DOI: 10.1016/j.jastp.2006.01.008.

Smith, E.J., and J.H. Wolfe. Observations of interaction regions and corotating shocks between one and five AU: Pioneers 10 and 11 . Geophys. Res. Lett., 3, 137-140, 1976, DOI: $10.1029 /$ GL003i003p00137.

Thayer, J.P., and J. Semeter. The convergence of magnetospheric energy flux in the polar atmosphere. J. Atmos. Sol. Terr. Phys., 66, 807-824, 2004, DOI: 10.1016/j.jastp.2004.01.035.

Thayer, J.P., J.F. Vickrey, R.A. Heelis, and J.B. Gary. Interpretation and modeling of the high-latitude electromagnetic energy flux. J. Geophys. Res., 100 (A10), 19715-19728, 1995, DOI: $10.1029 / 95 J A 01159$

Tsurutani, B.T., and W.D. Gonzalez. The cause of high-intensity long-duration continuous AE activity (HILDCAAs): interplanetary Alfvén wave trains. Planet. Space Sci., 35, 405, 1987.

Tsurutani, B.T., W.D. Gonzalez, A.L.C. Gonzalez, F. Tang, J.K. Arballo, and M. Okada. Interplanetary origin of geomagnetic activity in the declining phase of the solar cycle. J. Geophys. Res., 100, 21717-21733, 1995, DOI: 10.1029/95JA01476.

Tsurutani, B.T., W.D. Gonzalez, A.L.C. Gonzalez, F.L. Guarnieri, N. Gopalswamy, et al. Corotating solar wind streams and recurrent geomagnetic activity: a review. J. Geophys. Res., 111, A07S01, 2006. DOI: 10.1029/2005JA011273

Turner, N.E., W.D. Cramer, S.K. Earles, and B.A. Emery. Geoefficiency and energy partitioning in CIR-driven and CME-driven storms. J. Atmos. Sol. Terr. Phys., 71, 1023-1031, 2009, DOI: $10.1016 /$ j.jastp.2009.02.005.

Verkhoglyadova, O.P., B.T. Tsurutani, A.J. Mannucci, M.G. Mlynczak, L.A. Hunt, A. Komjathy, and T. Runge. Ionospheric VTEC and thermospheric infrared emission dynamics during corotating interaction region and high-speed stream intervals at solar minimum: 25 March to 26 April 2008. J. Geophys. Res., 116, A09325, 2011, DOI: 10.1029/2011JA016604. 
Verkhoglyadova, O.P., B.T. Tsurutani, A.J. Mannucci, M.G. Mlynczak, L.A. Hunt, and T. Runge. Variability of ionospheric TEC during solar and geomagnetic minima (2008 and 2009): external high speed stream drivers. Ann. Geophys., 31, 263-276, 2013, DOI: 10.5194/angeo-31-263-2013.

Verkhoglyadova, O.P., A.J. Mannucci, B.T. Tsurutani, M.G. Mlynczak, L.A. Hunt, R.J. Redmon, and J.C. Green. Localized thermosphere ionization events during the high-speed stream interval of 29 April to 5 May 2011. J. Geophys. Res. [Space Phys.], 120, 675-696, 2015, DOI: 10.1002/2014JA020535.

Vichare, G., A. Ridley, and E. Yigit. Quiet-time low latitude ionospheric electrodynamics in the non-hydrostatic Global
Ionosphere-Thermosphere Model. J. Atmos. Sol. Terr. Phys., 90, 161-172, 2012, DOI: 10.1016/j.jastp.2012.01.009.

Vourlidas, A. Mission to the Sun-Earth L5 Lagrangian point: an optimal platform for space weather research. Space Weather, 13, 197-201, 2015, DOI: 10.1002/2015SW001173.

Weimer, D.R. Improved ionospheric electrodynamic models and application to calculating Joule heating rates. J. Geophys. Res., 110, A05306, 2005, DOI: 10.1029/2004JA010884.

Zhang, Y., and L.J. Paxton. An empirical Kp-dependent global auroral model based on TIMED/GUVI FUV data, J. Atmos. Sol. Terr. Phys. 70, 1231-1242, 2008,

DOI: $10.1016 /$ j.jastp.2008.03.008.

Cite this article as: Verkhoglyadova $\mathrm{O}$, Meng X, Mannucci A, Tsurutani B, Hunt L, et al. Estimation of energy budget of ionospherethermosphere system during two CIR-HSS events: observations and modeling. J. Space Weather Space Clim., 6, A20, 2016,

DOI: $10.1051 /$ swsc $/ 2016013$. 